\title{
Shigella sonnei O-antigen inhibits internalisation, vacuole escape and inflammasome activation
}

Jayne L Watson ${ }^{1}$, Julia Sanchez-Garrido², Philippa J Goddard ${ }^{1,2}$, Vincenzo Torraca ${ }^{3}$, Serge Mostowy $^{3}$, Avinash R Shenoy ${ }^{2}$, Abigail Clements ${ }^{1 \#}$

${ }^{1}$ Department of Life Sciences and ${ }^{2}$ Department of Infectious Disease, MRC Centre for Molecular Bacteriology \& Infection, Imperial College London, London, United Kingdom, ${ }^{3}$ Department of Infection Biology, London School of Hygiene and Tropical Medicine, London, United Kingdom.

\# To whom correspondence should to be addressed: Dr Abigail Clements, MRC Centre for Molecular Bacteriology and Infection, Department of Life Sciences, Imperial College London, London SW7 2AZ; a.clements@imperial.ac.uk

Running Title: Interaction of Shigella sonnei and macrophages

\section{Abstract}

Two Shigella species, flexneri and sonnei, cause approximately $90 \%$ of bacterial dysentery worldwide. While $S$. flexneri is the dominant species in low-income countries, S. sonnei causes the majority of infections in middle and high-income countries. S. flexneri is a prototypic cytosolic bacterium; once intracellular it rapidly escapes the phagocytic vacuole and causes pyroptosis of macrophages, which is important for pathogenesis and bacterial spread. By contrast little is known about the invasion, vacuole escape and induction of pyroptosis during S. sonnei infection of macrophages. We demonstrate that $S$. sonnei causes substantially less pyroptosis in human primary monocyte-derived macrophages and THP1 cells. This is due to reduced bacterial uptake and lower relative vacuole escape, which results in fewer cytosolic S. sonnei and hence reduced activation of caspase- 1 inflammasomes. Mechanistically, the Oantigen, which in S. sonnei is contained in both the lipopolysaccharide and the capsule, was responsible for reduced uptake and the T3SS was required for vacuole escape. Our findings suggest that $S$. sonnei has adapted to an extracellular lifestyle by incorporating additional Oantigen into its surface structures compared to other Shigella species.

\section{Introduction}

Shigella are the causative agents of Shigellosis, infecting an estimated 125 million people annually. Children under five are most at risk with $61 \%$ of cases and $69 \%$ of deaths occurring 
in this age group (1). Closely related to Escherichia coli, the genus is made up of four species; S. flexneri, S. sonnei, S. dysenteriae and S.boydii. These are divided into serotypes based on the O-antigen (O-Ag) structure. S. flexneri and S. sonnei are responsible for the majority of infections however, dominance is highly dependent on the socioeconomic status of an area. $S$. flexneri is associated with poor water sanitation and hygiene in developing countries. In sub-Saharan Africa and Asia, S. flexneri accounts for $66 \%$ of cases and S. sonnei $24 \%$ of cases (2). However, in areas with good socioeconomic conditions and a high gross domestic product per capita, such as North America and Europe, S. sonnei is responsible for up to $80 \%$ of infections (3). Transitional countries that have recently undergone socioeconomic improvements show a shift from $S$. flexneri to $S$. sonnei as the dominant species (4-6). As a number of large populous countries undergo this shift (eg. Brazil, India, China), S. sonnei is emerging as an important pathogen.

The pathogenesis of $S$. sonnei is poorly understood and generally assumed to be similar to $S$. flexneri. The growing importance of $S$. sonnei has led to a re-evaluation of its pathogenesis and has revealed some important differences from $S$. flexneri. These include a novel adhesin, $(7,8)$, an antibacterial T6SS (9) and a group 4 capsule (G4C) which protects it from serummediated killing (10). Both species have a homologous T3SS that promotes secretion of effectors into host cells.

Unlike other Shigella species which contain multiple serotypes, there is only one S. sonnei serotype. The genes encoding biosynthesis and export of the O-Ag are encoded on the pSS virulence plasmid and were horizontally acquired from Plesiomonas shigelloides. In all other Shigella spp., these genes are located on the chromosome (11). The S. sonnei O-Ag is composed of two unusual sugars, 2-acetamido-2-deoxy-L-altruronic acid (L-AltNAcA) and 2acetamido-2-deoxy-L-fucose (FucNAc4N), not present in the O-Ag of other Shigella spp., or indeed in many bacteria (12). Importantly, the G4C of S. sonnei is also composed of the O-Ag polysaccharide, linked to an unknown lipid anchor rather than the lipid A-core as in the LPS (10). Therefore the surface of S. sonnei is covered with two O-Ag layers.

Pyroptotic cell death is considered an important component of $S$. flexneri pathogenesis, (13) allowing S. flexneri to escape macrophage-mediated killing, induce local inflammation and invade epithelial cells from the basolateral side (14). In the canonical pathway for caspase-1 activation and pyroptosis, NOD and leucine-rich repeat containing proteins with CARD or PYD (NLRCs or NLRPs), AIM2-like receptors or Pyrin protein can respond to pathogen- and/or danger-associated molecular patterns. This leads to the assembly of the sensor e.g. NLRP3 or NLRC4, and the adaptor protein, ASC, into a signalling platform, known as the inflammasome, which activates caspase-1 (15). In the non-canonical pathway, caspase-4 
directly senses and is activated by cytosolic LPS (16). Active-caspase-1 and active-caspase4 can cleave gasdermin-D (GSDMD) (17). Once cleaved, the N-terminal of GSDMD forms pores in the cell membrane to cause swelling and membrane rupture. Proinflammatory cytokines, IL-1 $\beta$ and IL-18, are also cleaved by active caspase- 1 into their mature forms and released $(18,19)$.

S. flexneri can activate the NLRC4 and NLRP3 inflammasomes (20). The T3SS needle and rod proteins (MxiH and Mxil, respectively) are recognised by hNaip/mNaip1 and mNaip2 proteins which interact with NLRC4 and promote caspase-1 activation $(21,22)$. NLRP3 senses decreased cytosolic potassium levels and activates caspase-1 (23). A T3SS effector, IpaH7.8, has been shown to be important for activation of both the NLRC4 and NLRP3 inflammasomes (20). In the case of Shigella, it is unclear whether pyroptosis benefits the host or the bacteria. S. flexneri is thought to use pyroptosis to escape the macrophage and infect epithelial cells. However, recent studies using Salmonella suggest that pyroptosis results in killing of bacteria by forming pore-induced intracellular traps (PITs) (24) or GSDMD targeting of bacterial membranes (25). It is currently unknown whether $S$. sonnei activates the same inflammasomes as S. flexneri and whether this is beneficial for the host or bacteria.

In this study, we demonstrate for the first time that $S$. sonnei induces caspase-1-dependent pyroptosis of human macrophages. However, we observed that equivalent bacterial inocula induced much less cell death for S. sonnei than S. flexneri. We show this is due to the O-Ag of $S$. sonnei which reduces internalisation and vacuole escape, resulting in less cytosolic bacteria. Our studies reveal an important role for the S. sonnei O-Ag in regulating bacterial interactions with macrophages, with one consequence being a reduction in inflammatory cell death.

\section{Results}

\section{S. sonnei induces less macrophage cell death than S. flexneri}

Previous research into the interactions of Shigella with macrophages has largely focused on $S$. flexneri, which robustly induces pyroptosis in macrophages (20). To investigate whether $S$. sonnei behaved in a similar manner, we infected primary human $\mathrm{CD} 14^{+}$monocyte derived macrophages (hMDMs) and measured the uptake of propidium iodide (PI), as an indicator of membrane damage that precedes pyroptosis. Unexpectedly, S. sonnei induced $50 \%$ less $\mathrm{PI}$ uptake than S. flexneri (figure 1a).

Similar experiments in PMA-differentiated THP1 cells recapitulated the reduced PI uptake during $S$. sonnei infection compared to $S$. flexneri (figure $1 \mathrm{~b}$ ), In addition, a lactate dehydrogenase $(\mathrm{LDH})$ release assay comparing lytic cell death showed $S$. sonnei induced 
less cell death than S. flexneri (figure 1c). To ensure reduced cell death was not a unique feature of the widely used S. sonnei strain 53G, we included a recent clinical isolate, S. sonnei 381 , alongside $S$. sonnei $53 G$ and compared these to two different $S$. flexneri strains M90T (serotype 5a) and 2457T (serotype 2a). Notably, both S. sonnei strains induced lower PI uptake in macrophages (figure $1 \mathrm{~b}$ ).

\section{There are fewer cytosolic S. sonnei than S. flexneri}

Induction of macrophage cell death by $S$. flexneri requires the bacteria to be cytosolic, which entails two steps: internalisation and vacuole escape. We hypothesised that differences in these processes between $S$. flexneri and $S$. sonnei might be responsible for the differences in cell death observed. To investigate why $S$. sonnei induced less cell death, we treated hMDMs or PMA-treated THP1 cells with $50 \mu \mathrm{M}$ Z-VAD-fmk, a pan-caspase inhibitor, to inhibit cell death (figure S1a) and performed a gentamicin protection assay to calculate the number of intracellular bacteria (figure $1 \mathrm{~d}$ and e). $S$. sonnei infected macrophages had reduced numbers of intracellular bacteria compared to $S$. flexneri.

As the earliest timepoint that can be measured in the gentamicin protection assay is 1 hour 40 min post infection, it is possible bacteria were already killed by this timepoint which would misrepresent the relative efficiency of internalisation (because internalised and killed bacteria would not be detected). To address this we enumerated intracellular bacteria by differential staining at 40 min post infection, which confirmed that fewer THP1 cells harboured intracellular bacteria when infected with $S$. sonnei than when infected with $S$. flexneri (figure 1f).

Internalised S. flexneri rapidly lyse the phagocytic/endosomal vacuole in order to access the cell cytosol and escape lysosomal degradation (26). To investigate how well S. sonnei escaped into the cytosol we used chloroquine ( $\mathrm{CHQ}$ ), an antibiotic that only accumulates in vacuoles at high enough concentrations to kill bacteria, allowing discrimination between cytosolic and vacuolar bacteria (27). S. sonnei showed a reduction in vacuole escape compared to $S$. flexneri (figure 1g). Taken together, these data indicated there are less cytosolic S. sonnei compared to $S$. flexneri at the same multiplicity of infection (MOI), which may result in the reduced macrophage cell death observed with $S$. sonnei. By increasing the S. sonnei $\mathrm{MOI}$ to obtain equivalent numbers of cytosolic bacteria to $S$. flexneri (figure $S 1 b$ ), $S$. sonnei and S. flexneri induced similar levels of cell death (figures $1 \mathrm{~h}$ and $\mathrm{j}$ ) and cell lysis (figure S1c). These findings confirm that cytosolic bacteria are required for induction of cell death in S. sonnei and $S$. flexneri and that $S$. sonnei does not access the cytosol as efficiently as $S$. flexneri. 
The T3SS is required for vacuole escape but not internalisation of S. sonnei

The T3SS of $S$. flexneri is required for bacteria to lyse the phagocytic vacuole and access the cytosol (28). Consistent with this, a $S$. sonnei T3SS mutant ( $\Delta m x i D)$ had an impaired ability to escape the vacuole (figure $2 a$ ) and reduced cell death measured by PI uptake (figure 2b) and $\mathrm{LDH}$ release (figure S1d). The S. sonnei T3SS was required to induce vacuole lysis and hence produce cytosolic bacteria.

It is unclear whether Shigella internalisation into macrophages is predominantly T3SSdependent invasion or phagocytic uptake. T3SS-mediated invasion of epithelial cells by $S$. flexneri triggers extensive membrane recruitment to engulf the bacteria. To visualise S. flexneri and S. sonnei uptake we performed scanning electron microscopy (SEM) on infected cells and were able to see membrane recruitment around attached $S$. flexneri but not $S$. sonnei (figure $2 \mathrm{c}$ and $\mathrm{d}$ ). As phagocytic uptake and T3SS-mediated invasion both involve membrane rearrangement these would be difficult to distinguish visually. Instead we performed gentamicin protection assays with wild type and T3SS mutants to quantify the number of intracellular bacteria in macrophages. Our experiments showed that in both hMDM and THP1 cells, internalisation into macrophages was T3SS-dependent for S. flexneri but not S. sonnei (figure $2 \mathrm{e}$ and $\mathrm{f}$ ). This suggested that the majority of $\mathrm{S}$. flexneri actively invaded macrophages, in contrast to $S$. sonnei, which were mainly internalised by phagocytic uptake.

\section{S. sonnei and S. flexneri induce similar pyroptosis pathways in infected macrophages}

Given that cytosolic bacteria induce cell death through inflammasome activation we characterised the inflammasome pathways activated by $S$. sonnei. As the $S$. flexneri inflammasome activators $\mathrm{MxiH}$, Mxil and IpaH7.8 proteins are $100 \%$ identical between $S$. sonnei $53 \mathrm{G}$ and $S$. flexneri M90T we hypothesised they would activate the NLRC4 inflammasome. At comparable levels of cytosolic bacteria, similar activation of caspase-1 and proteolytic cleavage of GSDMD and IL-18 were observed (figure 3a). The involvement of the inflammasome pathway was confirmed using ASC ${ }^{\text {mRFP }}$ THP1 cells which revealed that both bacteria induced comparable levels of cells with ASC-containing inflammasome foci during infection (figure $3 \mathrm{~b}$ and $\mathrm{c}$ ). Further, infected GSDMD-silenced THP1 cells (THPGSDMD-miR, validated in S2a and b) underwent reduced cell death, suggesting pyroptosis is the dominant type of cell death induced by S. sonnei and S. flexneri (figure 3d).

Cells deficient in caspase-4 showed reduced pyroptosis (figure 3e), however, loss of caspase1 almost completely abolished pyroptosis (figure $3 \mathrm{f}$, all knockout cells are validated in S2c-e), indicating the canonical pathway of pyroptosis predominates in S. sonnei and S. flexneri 
infected macrophages. Treatment with the NLRP3 inhibitor, MCC950 (29), did not markedly affect cell death (figure $3 g$ and validated in S2f), suggesting that NLRP3 plays a minor role in pyroptosis. ASC-deficient THP1 cells showed a partial reduction in cell death levels compared to WT THP1 cells (figure 3h). Taken together, these results are consistent with NLRC4 activation contributing to pyroptosis during $S$. sonnei infection of human macrophages, which is similar to previous reports for $S$. flexneri.

The T6SS and LVP instability do not account for reduced cell death caused by S. sonnei

All Shigella spp. harbour a large virulence plasmid (LVP) that encodes the T3SS, its effectors and additional important virulence factors. The LVP of $S$. sonnei is less stable than S. flexneri due to the evolution of different toxin:anti-toxin systems (30). We inserted an antibiotic resistance cassette onto the LVP to create a stabilised LVP and used this strain to test if LVP loss was affecting the amount of cell death that was induced. The LVP stabilised S. sonnei induced similar cell lysis as WT S. sonnei indicating that differences in plasmid retention was not responsible for the altered interaction with macrophages (figure 4a).

Even though the T6SS of $S$. sonnei has only been described to have anti-bacterial activity (9), T6SSs from other bacteria (e.g. Francisella tularensis $(31,32)$ ) have activity within macrophages. We therefore created a $S$. sonnei T6SS mutant $(\Delta t s s B)$ to determine if there was any contribution by the T6SS to cell death but found no difference in LDH release (figure $4 a)$, indicating that the T6SS was not responsible for the altered interaction with macrophages. Altogether, these results ruled out loss of LVP or a contribution by the T6SS in the reduced cell death observed for $S$. sonnei.

\section{The S. sonnei O-Antigen prevents internalisation into macrophages}

In S. sonnei the O-Ag is incorporated into the G4C as well as being attached to the lipid Acore of LPS (figure 4b). The incorporation of the O-Ag into LPS and G4C is genetically separable, which we exploited to investigate their respective roles in the interaction with macrophages. The G4C of $S$. sonnei reduces bacterial invasion of epithelial cells by impairing T3SS activity (10), and could therefore play a similar role in macrophage internalisation. We confirmed that $S$. sonnei $\triangle G 4 C$ invaded HeLa cells more efficiently (figure S3b). Uptake and pyroptosis induced by $S$. sonnei $\Delta G 4 C$ was statistically similar to wildtype bacteria, although we did observer slightly higher cell death with the $\Delta \mathrm{g} 4 \mathrm{c}$ mutant (figure $4 \mathrm{c}-\mathrm{d}$ ). This was consistent with predominantly phagocytic uptake of $S$. sonnei by macrophages.

We then deleted the O-Ag synthesis operon (genes wbgT to wbgZ) (33) to create a strain devoid of all O-Ag (both LPS and G4C linked) (figure $4 \mathrm{~b}$ ). This strain ( $\Delta \mathrm{O}-\mathrm{Ag}$ ) demonstrated 
wild type S. sonnei. By contrast, an LPS O-Ag deficient strain ( $\Delta$ waaL), which retains the G4C, showed equivalent internalisation as wild type S. sonnei (figure S3a). Therefore, the presence of the S. sonnei O-Ag per se, rather than specifically the O-Ag in the capsule or LPS, impedes macrophage internalisation and its complete removal enhances bacterial internalisation.

We have shown that $S$. sonnei cell death is T3SS-dependent due to the requirement for cytosolic bacteria. The T3SS tip accessibility has previously been shown to be enhanced upon removal of the G4C, and further exposed by removal of the O-Ag (10). We therefore hypothesised that the O-Ag was impeding T3SS mediated invasion. To test this we created a T3SS mutant in the O-Ag deficient strain $(\Delta \mathrm{O}-\mathrm{Ag} \Delta m x i \mathrm{D})$. In keeping with our hypothesis this strain had wild-type levels of internalisation (figure 4e), but impaired activation of cell death because it is unable to escape the vacuole. To further investigate the role of the O-Ag in shielding the T3SS we complemented the O-Ag mutant with either the S. sonnei O-Ag synthesis operon (pSS) or the S. flexneri 5a O-Ag synthesis and modification operons (pSf5a) (34-36) (figure 4e). Both complemented strains impeded internalisation of $S$. sonnei (figure 4f) and, as a consequence, reduced the level of cell death similar to those observed with wildtype S. sonnei (figure 4f). Interestingly, complementation with pSf5a produced a S. flexneri-like OAg ladder which migrated differently on SDS-PAGE than when expressed in S. flexneri. To determine if this was due to different modal length of O-Ag controlled by WzzB we introduced the $w z z B^{S f}$ onto the pSf5a complementation plasmid (pSf5a/wzzB). In this strain the modal length of the O-Ag resembled that of the wild type $S$. flexneri, however the levels of internalisation and cell death were not reduced to the levels of the wild type $S$. sonnei and instead resembled the levels of the O-Ag mutant.

\section{Discussion}

S. flexneri is known to induce pyroptosis in macrophages. This is considered a key step in the pathogenesis of Shigella as it allows bacteria to infect epithelial cells from the preferred basolateral side and leads to bacterial dissemination. In addition, pyroptosis creates an inflammatory response causing the recruitment of neutrophils, which disrupt the epithelial cell barrier and allows more Shigella to traverse the epithelial layer (37).

Here we present evidence that $S$. sonnei does not use the same mechanisms during infection as $S$. flexneri (summarised in figure 5). In line with previous reports, we found that $S$. flexneri induces rapid pyroptosis upon internalisation of infected macrophages $(20,22)$. However, $S$. sonnei induced markedly less macrophage cell death, which was the result of a decreased number of cytosolic bacteria through a combination of fewer internalised $S$. sonnei and impaired vacuole escape. The requirement for cytosolic bacteria in the induction of inflammasomes was consistent for both S. sonnei and S. flexneri. Additional host responses 
240 are also likely to be affected by the reduced number of cytosolic bacteria for $S$. sonnei 241 compared to S. flexneri.

242 Once $S$. sonnei and S. flexneri cytosolic numbers were normalised, pyroptosis proceeded via 243 similar pathways and to similar levels. For both species, cell death was predominantly 244 dependent on GSDMD and caspase-1, indicating the canonical inflammasome pathway is induced by Shigella. There may be a minor contribution to cell death for the non-canonical pathway as immunoblots indicated that caspase-4 was activated by infection of both $S$. sonnei and S. flexneri, and caspase-4 deficiency or NLRP3 inhibition led to less pyroptosis over time than control cells. However, this difference was minor compared to that observed for ASC or caspase-1-deficient cells. NLRC4 has a caspase-recruitment and activation domain (CARD) which can enable its interaction with, and activation of caspase-1 directly, bypassing the need for ASC (38-40). This suggests that the NLRC4 inflammasome has a prominent role in the cell death of S. sonnei-infected THP1 cells. These results are in line with those shown previously for S. flexneri, which suggest both the NLRP3 and NLRC4 inflammasomes are involved in S. flexneri-mediated macrophage death (20).

Interestingly, $\mathrm{S}$. sonnei was able to reduce internalisation into macrophages in an O-Ag dependent manner. The O-Ag contributes to host immune evasion and its role in evasion of complement mediated killing is well characterised (41). There are also examples of O-Ags affecting cellular interactions including impeding recognition and internalisation by epithelial cells (Salmonella Typhimurium (42)) and macrophages (Burkholderia cenocepacia (43)). The modal length of the O-Ag from Salmonella Typhimurium or S. flexneri serotype 2a is important for T3SS-mediated invasion into macrophages and epithelial cells respectively (44). Similarly, glucosylation of the S. flexneri serotype $5 \mathrm{a}$ O-Ag which reduces the O-Ag length by half enhances its invasiveness (45).

Unexpectedly, in our study the internalisation of S. sonnei into macrophages was independent of its T3SS. This is in contrast to S. flexneri which exhibits significant T3SS-mediated invasion into macrophages. This suggests that macrophage internalisation is a combination of bacteriadriven invasion and phagocytic uptake for S. flexneri, while $S$. sonnei internalisation is almost exclusively due to phagocytic uptake. The S. sonnei O-Ag is incorporated into both the G4C and the LPS of $S$. sonnei. Only when all the O-Ag layers of $S$. sonnei are removed is S. sonnei able to efficiently invade macrophages. The accessibility of IpaB was previously shown to increase upon removal of the G4C and a further increase was observed for an O-Ag-deficient 272 strain indeed suggesting the lipid A-core linked O-Ag also contributes to shielding of the T3SS 273 (10). The ability of the serotype 5a O-Ag synthesis and modification operon from S. flexneri to prevent internalisation of the O-Ag deficient S. sonnei indicates the composition of the 
275 saccharides are not important for this phenotype. Furthermore, the inability of the S. flexneri

276 O-Ag when regulated by wzzB to complement for internalisation of cell death suggests the

277 modal length of the O-Ag is important. However, this strain also produced a low amount of O-

$278 \mathrm{Ag}$ and we cannot discount this as the reason for the failure to complement. Our data, and

279 previously published data regarding the accessibility of the T3SS, supports the conclusion that

280 the O-Ag acts as a physical barrier to T3SS-mediated invasion rather than being anti-

281 phagocytic.

282 The results presented here, combined with previous investigations, indicate that $S$. sonnei and

283 S. flexneri use different infection mechanisms. These mechanisms are also different from

284 related Gram-negative enteric pathogens such as Salmonella or EPEC, which also activate

285 distinct inflammasome pathways in human macrophages (46-49). Increasing evidence points

286 to $S$. sonnei being more adapted to an extracellular lifestyle as, compared to $S$. flexneri, it

287 invades epithelial cells and macrophages poorly. This may partly explain the dominance of $S$.

288 sonnei in developed countries where improved living conditions, including reduced

289 overcrowding and hence person-person spread of pathogens, fails to lower S. sonnei infection

290 rates. These studies highlight that further investigation into $S$. sonnei is required in order to 291 implement appropriate measures to reduce infection rates. 


\section{Bacterial strains and growth}

296 Unless otherwise stated, all Shigella strains (Table S1) were routinely grown in tryptone soya broth (TSB) at $37^{\circ} \mathrm{C}$ with shaking at 200 RPM. Antibiotic selection was used when necessary as follows: $100 \mu \mathrm{g} / \mathrm{mL}$ ampicillin (Amp), $50 \mu \mathrm{g} / \mathrm{mL}$ kanamycin (Kn), $12.5 \mu \mathrm{g} / \mathrm{mL}$ chloramphenicol $(\mathrm{Cm}), 100 \mu \mathrm{g} / \mathrm{mL}$ erythromycin, $50 \mu \mathrm{g} / \mathrm{mL}$ streptomycin $(\mathrm{Sm}), 10 \mu \mathrm{g} / \mathrm{mL}$ gentamicin (Gm).

\section{Cloning and mutagenesis}

302 S. sonnei LVPStabile, S. sonnei $\Delta$ waaL, S. sonnei $\Delta t s s B$ and S. sonnei $\Delta \mathrm{O}-\mathrm{Ag}$ strains were 303 constructed as follows, primer sequences are in Table S2. For S. sonnei LVP Stabile nt 8293683715 and nt 83716-84215 were amplified using primers 1 and 2, and 3 and 4 . The chloramphenicol cassette was amplified from pKD3 using primers 21 and 22. Overlapping PCR was used to construct the mutagenesis fragment consisting of 82936-83715-Cm-8371684215, (note the P1-P2 fragment was inserted in the opposite orientation). This fragment was further amplified by PCR with primers 1 and $4.2 \mu \mathrm{g}$ of PCR product was electroporated into S. sonnei $53 \mathrm{G}+$ pKD46 induced with $1 \mathrm{mM}$ L-arabinose for $45 \mathrm{~min}$ to express lambda red recombinase genes. The electroporation was plated on TSB supplemented with $\mathrm{Cm}$. Genomic insertion of cat was verified by PCR using primers 5 and 22

For $S$. sonnei $\Delta t s s B 500 \mathrm{bp}$ fragments flanking $t s s B$ were amplified using primers 6 and 7 , and 8 and 9. The kanamycin cassette was amplified from pKD4 using primers 21 and 22. Overlapping PCR was used to construct the mutagenesis fragment consisting of 5 ' tss $B$-kan3' tss $B$. This fragment was further amplified by PCR with primers 6 and 9.2 ug of PCR product was electroporated into $S$. sonnei $53 \mathrm{G}+$ pKD46 induced with $1 \mathrm{mM} \mathrm{L-arabinose} \mathrm{for} 45 \mathrm{~min}$ to express lambda red recombinase genes. The electroporation was plated on TSB supplemented with Kn. Genomic insertion of kan was verified by PCR using primers 10 and 22.

For S. sonnei $\Delta$ waaL $500 \mathrm{bp}$ fragments flanking waaL were amplified using primers 11 and 12 , and 13 and 14. The kanamycin cassette was amplified from pKD4 using primers 21 and 22. Overlapping PCR was used to construct the mutagenesis fragment consisting of 5' waaL-kan3' waaL. This construct and pSEVA612S were digested with BamHI and EcoRI, ligated and transformed into $E$. coli CC118- $\lambda$ pir. The resulting plasmid pSEVA $\triangle$ waaL-Kn was conjugated into S. sonnei 53G. Briefly, $20 \mu \mathrm{l}$ helper E. coli $1047 \mathrm{pRK} 2013$ was incubated for $2 \mathrm{~h}$ at $37^{\circ} \mathrm{C}$

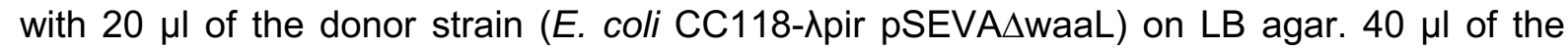
receiver strain (S. sonnei $53 G$ with PACBSR) was added and the plate incubated for $4 \mathrm{~h}$ at 
$37^{\circ} \mathrm{C}$. Conjugants were selected on TSB agar supplemented with $\mathrm{Gm}$ and Sm. Individual colonies were grown in TSB supplemented with Sm and 0.4\% (w/v) L-arabinose (Sigma) for $8 \mathrm{~h}$ to induce expression of the I-Scel endonuclease from pACBSR, and plated on Kn plates. Genomic deletion of waaL was verified by PCR using primers 15 and 22. The strains were passaged several times in liquid TSB to remove pACBSR and bacteria sensitive to Sm were selected.

S. sonnei $\Delta \mathrm{O}-\mathrm{Ag}$ mutant was constructed by amplifying $500 \mathrm{bp}$ fragments upstream of $w b g T$ and downstream of wbgZ using primers 16 and 17 and 18 and 19. The kanamycin cassette was amplified from pKD4 using primers 21 and 22. Overlapping PCR was used to construct the mutagenesis fragment consisting of 5' wbgT -kan-3' wbgZ. This construct and pSEVA612S were digested with BamHI and EcoRI, ligated and transformed into E. coli CC118- $\lambda$ pir. The resulting plasmid pSEVA $\triangle \mathrm{O}-\mathrm{Ag}-\mathrm{Kn}$ was conjugated into S. sonnei $53 \mathrm{G}$ as described above. Genomic deletion of $\triangle \mathrm{O}$-Ag was verified by PCR using primers 20 and 22 .

Complementation vectors were constructed using standard molecular biology techniques. The $53 \mathrm{~g}$ O-Ag operon was amplified with primers 23 and 24 . The PCR product and pSEVA471 were digested with $\mathrm{BamHI}$ and ligated to create $\mathrm{PO}-\mathrm{Ag}^{\mathrm{Ss}}$. The M90T gtr operon was amplified with primers 25 and 26 . The PCR product and pSEVA471 were digested with Kpnl and BamHI and ligated to create pSEVA471-gtr. The M90T O-Ag operon was amplified with primers 27 and 28. The PCR product and pSEVA471-gtr were digested with BamHI and $\mathrm{Xbal}$ and ligated to create POAg ${ }^{\text {Sf5a }}$. The M90T wzzB gene was amplified with primers 29 and 30 . The PCR product and $\mathrm{pOAg}^{\mathrm{sf5a}}$ were digested with $\mathrm{Kpnl}$ and ligated to create $\mathrm{pOAg}^{\mathrm{Sf5a} / \mathrm{wzzB}}$. All complementation constructs include predicted promoters and terminators.

\section{Cell culture and infection}

THP-1 cells were maintained in Roswell Park Memorial Institute (RPMI) medium supplemented with $10 \%$ heat-inactivated foetal bovine serum (FBS), $5 \mathrm{mM}$ Hepes, $5 \mathrm{mM}$ sodium pyruvate, $100 \mu \mathrm{g} / \mathrm{ml}$ penicillin and $100 \mu \mathrm{g} / \mathrm{ml}$ streptomycin. Cells were seeded at $7.5 \times 10^{5}$ cells $/ \mathrm{ml} 72$ hours prior to infection in complete RPMI $+100 \mathrm{ng} / \mathrm{ml}$ phorbol-12myristate-13-acetate (PMA). 24 hours prior to infection, media was replaced with phenol-red free, PMA-free complete RPMI medium. HeLa cells were maintained in Dulbecco's Modified Eagle's Medium (DMEM) (1000 mg/L glucose) supplemented with $10 \%$ FBS. Cells were seeded at $1 \times 10^{5}$ cells $/ \mathrm{ml}, 24$ hours prior to infection. All cell lines were incubated at $37^{\circ} \mathrm{C}, 5 \%$ $\mathrm{CO}_{2}$. Cells were infected with indicated multiplicity of infection (MOI) and centrifuged for 10 mins at $600 \mathrm{xg}$ to synchronise infection. At 30 minutes post centrifugation, $\mathrm{Gm}(150 \mu \mathrm{g} / \mathrm{ml})$ was added to directly to wells for the remainder of the experiment. Where indicated, inhibitors, 
Z-VAD-fmk (50 $\mu \mathrm{M}$; R\&D) or MCC950 (5 $\mu \mathrm{M}$; Tocris Bioscience), were added to cells 1 hour prior to infection. To induce NLRP3-driven caspase-1 activation, cells were primed with ultrapure O111:B4 LPS (250 ng/mL; Invivogen) for 3 hours and then treated with nigericin (20 $\mu \mathrm{M}$; Sigma) for 45 minutes. To induce caspase-4 activation, unprimed cells were transfected with LPS $(5 \mu \mathrm{g} / \mathrm{ml})$ using Lipofectamine 2000 (1\% v/w; Invitrogen).

HeLa infected cells were washed and fixed in $2.5 \%$ glutaraldehyde for analysis by scanning electron microscopy at an accelerating voltage of $25 \mathrm{kV}$ using a JEOL JSM-5300 scanning electron microscope [JEOL (UK), Herts, UK].

\section{Generation of cell lines}

The THP1 GSDMD ${ }^{\text {miR }}$ cell line was generated previously as described (50), THP1 Casp1 KO, THP1 Casp4 KO, and THP1 ASC KO were all kindly provided by Veit Hornung (51).

\section{Isolation of primary human monocyte derived macrophages}

Leukocytes cones were obtained from the NHS blood and transfusion service (from anonymous healthy donations), as previously described (46). Blood from each donor was diluted 1:4 with PBS, transferred into a LeucoSep tube (Greiner Bio-One) and centrifuged at $1000 \mathrm{xg}, 20$ mins at RT (slow acceleration and deceleration to prevent disturbance of the layers) to obtain the buffy coat containing white blood cells. This was separated and washed three times with RPMI. Cell were washed with MACS buffer $(50 \mathrm{mg} / \mathrm{ml} \mathrm{BSA}, 2 \mathrm{mM}$ EDTA in PBS). CD14+ cells were isolated by MACS using biotinylated anti-CD14+ antibody and antibiotin microbeads according to the manufacture's protocol (Miltenyi Biotec). Monocytes were cultured in complete RPMI plus $20 \mathrm{ng} / \mathrm{ml}$ recombinant human M-CSF for 7 days to promote differentiation into hMDMs. Media was replaced with complete RPMI lacking antibiotics and M-CSF 24 hours prior to infection.

\section{Internalisation and vacuole-escape assays}

To prevent cell death, cells were treated with Z-VAD-fmk $(50 \mu \mathrm{M}) 1$ hour prior to infection. Cells were infected with bacteria as described above. For internalisation assays, cells were washed with serum-free RPMI and lysed with TritonX-100 (0.5\%) at 1.5 hours post infection. For vacuole-escape assays, cells were treated $30 \mathrm{~min}$ post-infection with either $200 \mu \mathrm{g} / \mathrm{ml}$ chloroquine and $150 \mu \mathrm{g} / \mathrm{ml}$ gentamicin or $150 \mu \mathrm{g} / \mathrm{ml}$ gentamicin alone for $1 \mathrm{~h}$ and then lysed with TritonX-100 (0.5\%). Serial dilutions were performed, plated on LB agar and incubated overnight at $37^{\circ} \mathrm{C}$.

\section{PI uptake assays}


Cells and bacterial strains were prepared as described above. Prior to infection, cells were supplemented with $5 \mu \mathrm{g} / \mathrm{ml}$ Propidium iodide (PI; Invitrogen). For timecourse assays, fluorescence was measured at $630 \mathrm{~nm}$ every 10 mins with POLARStar 623 Omega plate reader (BMG Labtech) (52). Uninfected controls treated with TritonX-100 (0.05\%) were used to calculate percentage uptake.

\section{LDH assays}

Infections were performed as described above. At 3 hours post infection, supernatants were harvested. An LDH assay was performed as per kit instructions (CytoTox 96® NonRadioactive Cytotoxicity Assay, Promega). Absorbance was measure at $490 \mathrm{~nm}$ and values are expressed as percentage of $100 \%$ lysis control. All values are normalised to uninfected control.

\section{Immunoblots}

Infections were performed as described previously, except prior to infection cells were washed with PBS and infections were done in OptiMEM + $5 \mathrm{mM}$ Na pyruvate. Supernatants were precipitated in acetone $(1: 4 \mathrm{v} / \mathrm{v})$ overnight at $-20^{\circ} \mathrm{C}$, acetone was aspirated, and samples left to air dry. Cells were lysed in RIPA buffer (120 mM Tris pH 8.0, $300 \mathrm{mM} \mathrm{NaCl}, 2 \% \mathrm{NP}-40,1 \%$ $\mathrm{Na}$ deoxycholate, $2 \mathrm{mM}$ EDTA) supplemented with complete protease inhibitor and $1 \mathrm{mM}$ PMSF. Laemmli buffer and 5\% 2-mercaptoethanol were added to lysates. Precipitated supernatants were re-suspended in respective cell lysates to create pooled samples. Mouse anti-hcaspase-1 (AdipoGen), mouse anti-caspase-4 (Santa Cruz biotechnology), goat antihIL1 $\beta$ (R and D systems), rabbit anti-hIL18 (MBL international) were used at 1:1000 dilution and mouse anti-hGSDMD (Santa Cruz Biotechnology) was used at 1:500.

\section{Immunofluorescence microscopy}

Cells were seeded and infected as described previously. For calculation of percentage of THP1 cells infected, in/out staining was performed as follows. 40 minutes post addition of bacteria, $T=0$, cells were washed three times with cold PBS. Rabbit anti-sonnei (1:100) (phase 1 and 2 sera, Fisher Scientific) or rabbit anti-flexneri (1:500) (serotype 5a sera, PHE) diluted in $2 \%$ Bovine serum albumin (BSA):PBS were added to cells. Cells were incubated with antibodies on ice for 30 minutes. Following this, cells were washed with cold PBS, and incubated on ice with donkey anti-rabbit-Alexa594. (1:500, 2\% BSA:PBS). Cells were fixed with $2 \%$ paraformaldehyde (PFA) diluted in PBS for 20 minutes, washed in PBS, and neutralised with $50 \mathrm{mM} \mathrm{NH}_{4} \mathrm{Cl}$. $0.1 \%$ Triton-X 100 was added to cells for 8 minutes to permabilise. DAPI (1:1000, 2\% BSA:PBS) (Invitrogen) and phalloidin-Alexa647 (1:100) (Invitrogen) were incubated with cells. Coverslips were mounted onto slides with ProLong® 
429 Gold Antifade Mountant and visualised using Zeiss Axio Observer Z1 microscope. For 430 counting of ASC foci, ASC ${ }^{\text {mRFP }}$ cells (46) were infected as described, washed in PBS at 3 hours

431 post infection and fixed. The protocol was then continued as described above.

\section{LPS preparation and visualisation}

433 Crude LPS was prepared as follows. $1.5 \mathrm{ml}$ of overnight culture was centrifuged, resuspended 434 in laemmli buffer and boiled for $5 \mathrm{~min}$. Proteinase $\mathrm{K}(1 \mathrm{mg} / \mathrm{ml})$ was added and incubated for 2 $435 \mathrm{~h}$ at $56^{\circ} \mathrm{C}$. 2-mercaptoethanol $(5 \%)$ was added and samples were boiled for $5 \mathrm{~min}$ and $5 \mathrm{ul}$ of each sample separated by $12 \%$ SDS-PAGE. The gel was either transferred to PVDF and incubated with S. flexneri serotype 5a antibody (PHE) or S. sonnei phase I antibody (Abcam), followed by anti-rabbit HRP and developed by chemiluminescence, or fixed and silver stained as previously described (53).

\section{Statistical Analysis}

441 The number of independent repeats performed for each experiment is indicated (by $n$ ) in the figure legend. One-way ANOVA or Student's t-test was performed to compare means as implemented in GraphPad prism 8. Errors bars represent standard error of the mean throughout.

\section{Acknowledgements}

ARS would like to acknowledge funding from the MRC (MR/P022138/1). JLW is the recipient of an MRC Centre for Molecular Bacteriology and Infection (CMBI) PhD scholarship as part of the CMBI Centre Award MR/J006874/1.

\section{Conflict of Interests}

The authors declare that they have no conflict of interest

Figure Legends

Figure 1. S. sonnei induces less pyroptosis of macrophages than S. flexneri

(A) Primary human monocyte-derived macrophage (hMDMs) were infected with the indicated Shigella strains and cell death was measured by PI uptake at $3 \mathrm{~h}$ post infection. ${ }^{*} \mathrm{p}<0.05$ by paired Student's $t$ test, $n=4$ independent repeats from two donors.

(B and C) THP1 cells were infected with the indicated wild type Shigella strains. Cell death was measured by PI uptake over a $3 \mathrm{~h}$ time-course and plotted as Area Under the Curve 
460 (AUC), $n=3$ (B) or $\mathrm{LDH}$ release at $3 \mathrm{~h}$ post infection, $n=9(\mathrm{C}) .{ }^{* * * *} \mathrm{p}<0.0001$ by one-way

461 ANOVA with Tukey's multiple comparisons test $(B)$ or ${ }^{* * * *} p<0.0001$ by paired Student's $t$ test 462 (C).

463 (D) hMDMs and (E) THP1 cells were infected with S. sonnei or S. flexneri and gentamicin464 protected intracellular bacteria determined by CFU enumeration. (D) ${ }^{*} p<0.05$ by paired 465 Student's $t$ test, $n=4$ independent repeats from two donors and $(E){ }^{* * *} p<0.001$ by paired 466 Student's t test, $n=11$.

467 (F) Immunofluorescence microscopy was used to visualise intracellular/extracellular bacteria 468 and the percentage of infected THP1 cells calculated. ${ }^{* *} p<0.01$ by paired Student's $t$ test, $n=3$.

469 (G) THP1 cells were infected with S. sonnei or S. flexneri and subsequently treated with 470 gentamicin alone or gentamicin and choloroquine to determine the percentage of cytosolic 471 bacteria that have escaped the vacuole. ${ }^{* * *} p<0.001$ by paired Student's $t$ test, $n=4$.

472 (H) THP1 cells were infected with S. sonnei at an MOI of 20 or S. flexneri at an MOI of 5. Cell 473 death was measured by PI uptake over a $3 \mathrm{~h}$ time-course and plotted as Area Under the Curve 474 (AUC). ns, non-significant by paired Student's t test, $n=3$.

475 (I) hMDMs were infected with S. sonnei at an MOI of 40 or S. flexneri at an MOI of 10 and PI 476 uptake was measured at $3 \mathrm{~h}$ post infection. $\mathrm{ns}$, non-significant by paired Student's t-test, $\mathrm{n}=4$ independent repeats from two donors.

Figure 2.

480 (A) THP1 cells were infected with S. sonnei or S. flexneri and their respective T3SS mutants 481 and subsequently treated with gentamicin alone or gentamicin and choloroquine to determine the percentage of cytosolic bacteria that have escaped the vacuole. ${ }^{* * *} p<0.001$ by one-way ANOVA with Tukey's multiple comparisons test, $n=4$.

(B) THP1 cells were infected with wild type S. sonnei and S. flexneri and their respective T3SS mutants. Cell death was measured by PI uptake over a $3 \mathrm{~h}$ time-course and plotted as Area 486 Under the Curve (AUC). ns, non-significant, ${ }^{* * * *} p<0.0001$ by one-way ANOVA withTukey's 487 multiple comparisons test, $\mathrm{n}=3$.

488 (C and D) HeLa cells were infected with wild type S. sonnei and S. flexneri for 10 min before 489 being washed and fixed for SEM analysis. Arrows indicate bacteria attached to the cell 490 surface. 
491 (E) THP1 cells and (F) hMDMs were infected with wild type or T3SS-deficient S. sonnei and

492 S. flexneri and gentamicin-protected internalised bacteria were determined by CFU 493 enumeration. ns, non-significant, ${ }^{*} p<0.05$, ${ }^{* *} p<0.01$ by one-way ANOVA with Tukey's multiple comparisons test, $(E) n=4$ and $(F) n=4$ independent repeats from two donors.

Figure 3. S. sonnei and S. flexneri induce similar pyroptotic pathways when normalised for numbers of cytosolic bacteria.

498 (A) Immunoblots were performed on Shigella-infected THP1 cells to visualise cleavage of 499 Caspase-1, GSDMD, and IL-18 at $3 \mathrm{~h}$ post infection.

500 (B and C) ASC ${ }^{\text {mRFP }}$ THP1 cells were infected with fluorescent Shigella (green) at the indicated MOIs and after $3 \mathrm{~h}$ infection ASC foci formation visualised (red). DAPI staining was used to visualise DNA (blue). Representative micrographs for each strain are shown. The area indicated by the white box in the merged panel is enlarged to show single ASC foci in infected cells, and lack of ASC foci in uninfected cells. ASC foci formation was enumerated in (C). ns, non-significant, ${ }^{* *} p<0.01$ by One-way ANOVA, $n=4$.

(D) THP1 cells expressing non-targeting miRNA or GSDMD-targeting miRNA were infected with S. sonnei (MOI of 20) or S. flexneri (MOI of 5) for $3 \mathrm{~h}$. Cell death was measured by PI uptake over a $3 \mathrm{~h}$ time-course and plotted as Area Under the Curve (AUC). ${ }^{* * *} \mathrm{p}<0.0001$ by one-way ANOVA with Tukey's multiple comparisons test, $n=3$.

(E) Control THP1 cells (Ctrl) and THP1 cells deficient for Caspase-4 (Casp4 KO) were infected with S. sonnei (MOI 20) or S. flexneri (MOI 5). Cell death was measured by PI uptake over a $3 \mathrm{~h}$ time-course and plotted as Area Under the Curve (AUC). ${ }^{* * *} p<0.001$ and ${ }^{* * * *} p<0.0001$ by one-way ANOVA with Tukey's multiple comparisons test, $n=3$.

(F) Control THP1 cells (Ctrl) and THP1 cells deficient for Caspase-1 (Casp1 KO) were infected with S. sonnei (MOI 20) or S. flexneri (MOI 5). Cell death was measured by PI uptake over a $3 \mathrm{~h}$ time-course and plotted as Area Under the Curve (AUC). ${ }^{* * *} \mathrm{p}<0.0001$ by one-way ANOVA with Tukey's multiple comparisons test, $n=3$.

(G) THP1 cells left untreated or treated with $5 \mu \mathrm{M}$ MCC950 were infected with S. sonnei (MOI of 20) or S. flexneri (MOI of 5) for $3 \mathrm{~h}$. Cell death was measured by PI uptake over a $3 \mathrm{~h}$ timecourse and plotted as Area Under the Curve (AUC). ${ }^{* * * *} p<0.0001$ by one-way ANOVA with Tukey's multiple comparisons test, $\mathrm{n}=4$.

(H) THP1 cells and ASC-deficient THP1 cells were infected with S. sonnei (MOI of 20) or S. flexneri (MOI of 5 ) for $3 \mathrm{~h}$. Cell death was measured by $\mathrm{PI}$ uptake over a $3 \mathrm{~h}$ time-course and 
plotted as Area Under the Curve (AUC). ${ }^{* * *} p<0.0001$ by one-way ANOVA Tukey's multiple comparisons test, $\mathrm{n}=3$.

\section{Figure 4. Presence of the S. sonnei O-Ag reduces cells death and internalisation.}

(A) THP1 cells were infected with wild type S. sonnei, S. sonnei LVPStabile or S. sonnei $\Delta$ T6SS and cell death was measured by LDH release at $3 \mathrm{~h}$ post infection. Ns, non-significant by oneway ANOVA with Tukey's multiple comparisons test, $n=3$.

(B) S. sonnei has O-Ag attached to the cell surface in two different forms, as the conventional O-Ag attached to the Lipid A-core of the LPS, and as an O-Ag capsule attached to the cell surface by an unknown lipid anchor. $S$. sonnei $\triangle \mathrm{G} 4 \mathrm{C}$ has only the O-Ag incorporated into the LPS, S. sonnei $\triangle \mathrm{O}-\mathrm{Ag}$ has neither G4C or LPS O-Ag and S. sonnei $\triangle$ waaL has only the G4C $\mathrm{O}-\mathrm{Ag}$.

(C) THP1 cells were infected with wild type S. sonnei, S. sonnei $\Delta \mathrm{G} 4 \mathrm{C}$ or S. sonnei $\Delta \mathrm{O}-\mathrm{Ag}$ and gentamicin-protected internalised bacteria were determined by CFU enumeration. ${ }^{*} p<0.05$ by one-way ANOVA with a mixed effects model with Sidak's multiple comparisons test, $\mathrm{n}=10$ (wild type) and 7 (mutants).

(D) THP1 cells were infected with wild type S. sonnei, S. sonnei $\Delta \mathrm{G} 4 \mathrm{C}$ or S. sonnei $\Delta \mathrm{O}-\mathrm{Ag}$. Cell death was measured by PI uptake over a $3 \mathrm{~h}$ time-course and plotted as Area Under the Curve (AUC). ${ }^{* * *} p<0.0001$ by one-way ANOVA with Tukey's multiple comparisons test, $n=4$.

(E) Crude LPS was purified from the indicated S. sonnei and S. flexneri strains, separated by $12 \%$ SDS-PAGE and visualised by modified silver stain.

(F) THP1 cells were infected with wild type S. sonnei, S. sonnei $\Delta \mathrm{O}-\mathrm{Ag}$ or S. sonnei $\Delta \mathrm{O}-\mathrm{Ag}$ complemented with the S. sonnei O-Ag (pO-Ag ${ }^{\mathrm{Ss}}$ ), the S. flexneri O-Ag (pO-Ag ${ }^{\mathrm{Sf5a}}$ ) or the $S$. flexneri O-Ag and WzzB (pO-Ag ${ }^{\mathrm{Sf5a} / \mathrm{wzzB}}$ ) and gentamicin-protected internalised bacteria were determined by CFU enumeration. ${ }^{*} p<0.05$, ${ }^{* *} p<0.01$ by one-way ANOVA with Tukey's multiple comparisons test, $\mathrm{n}=4$.

(G) THP1 cells were infected with wild type S. sonnei, S. sonnei $\Delta \mathrm{O}-\mathrm{Ag}$, S. sonnei $\Delta \mathrm{O}-\mathrm{Ag}+$ $\mathrm{pO}-\mathrm{Ag}^{\mathrm{Ss}}$ or S. sonnei $\Delta \mathrm{O}-\mathrm{Ag}+\mathrm{pO}-\mathrm{Ag}^{\mathrm{Sf5}}$. Cell death was measured by PI uptake over a $3 \mathrm{~h}$ time-course and plotted as AUC. ${ }^{* * *} p<0.0001$ by one-way ANOVA with Tukey's multiple comparisons test, $n=4$. 

with macrophages.

557 S. flexneri uses T3SS-mediated invasion and vacuole rupture to reach the cytosol where it 558 induces inflammasome activation, GSDMD pores and pyroptosis. S. sonnei does not invade macrophages using its T3SS and only becomes intracellular through phagocytosis. Combined with a decreased ability to escape the vacuole this reduces the number of cytosolic bacteria and leads to reduced inflammasome activation. The T3SS becomes accessible in S. sonnei $\Delta \mathrm{O}-\mathrm{Ag}$ allowing the bacteria to invade macrophages and reach the cytosol where they can activate the inflammasome and cause pyroptosis.

Figure S1.

(A) THP1 cells were left untreated or treated with $50 \mu \mathrm{M} Z$-VAD-fmk one hour prior to infection Area Under the Curve (AUC). ${ }^{* * *} \mathrm{p}<0.0001$ by unpaired Student's $t$ test, $n=3$

(B) THP1 cells were infected with the indicated MOIs of S. sonnei or S. flexneri and the number of cytosolic CFU were enumerated after treating cells with gentamicin and chloroquine to kill extracellular and vacuolar bacteria. Ns, non-significant by one-way ANOVA, $n=4$. mutants. Cell death was measured by LDH release at $3 \mathrm{~h} .{ }^{* *} p<0.01,{ }^{* * *} p<0.001,{ }^{* * *} p<0.0001$ by one-way ANOVA, $n=3$.

(D) THP1 cells were infected with S. sonnei or S. flexneri and the respective T3SS mutants.

Figure S2. Phenotypic confirmation of THP1 cell lines

(A) GSDMD ${ }^{\text {miR }}$ and Control ${ }^{\mathrm{miR}}$ THP1 cells were treated with LPS and nigericin. Cell death was measured by PI uptake over a $3 \mathrm{~h}$ time-course and plotted as Area Under the Curve (AUC). ${ }^{* * * *} p<0.0001$ by one-way ANOVA, $n=3$.

(B) Representative immunoblots show silencing of GSDMD, $\beta$-actin serves as a loading control.

584 (C and D) Control THP1 cells and THP1 cells deficient for caspase-1, ASC or caspase-4 were 
NLRP3 activation (E). Cell death was measured by PI uptake over a $3 \mathrm{~h}$ timecourse and plotted as AUC. ${ }^{* * * *} p<0.0001$ by one-way ANOVA, $n=3$.

(E) Representative immunoblot shows knockout of Caspase-1, ASC or Caspase-4 loss in the appropriate cell line. $\beta$-actin serves as a loading control.

(F) LPS + nigericin was added to THP1 cells incubated with or without $5 \mu \mathrm{M}$ MCC950. Cell death was measured by PI uptake over a $3 \mathrm{~h}$ timecourse and plotted as AUC. ${ }^{* * * *} \mathrm{p}<0.0001$ by one-way ANOVA, $n=4$.

\section{Figure S3. Characterisation of S. sonnei O-Ag mutants}

(A) HeLa cells were infected with S. sonnei, S. sonnei $\Delta \mathrm{g} 4 \mathrm{c}$ or S. flexneri and gentamicin protected internalised bacteria were determined by CFU enumeration. ${ }^{*} p<0.05$ by one-way ANOVA, $n=3$.

(B) THP1 cells were infected with wild type S. sonnei or S. sonnei $\Delta$ waaL and gentamicinprotected internalised bacteria were determined by CFU enumeration. Ns, non-significant by one-way ANOVA, $n=5$

(C and D) Crude LPS was purified from the indicated S. sonnei and S. flexneri strains and the presence of the S. sonnei O-Ag (C) or S. flexneri 5a O-Ag (D) was detected using serotype specific antibodies.

\section{References}

1. Ashkenazi S. 2004. Shigella infections in children: New insights, vol 15, p 246-252.

2. Livio S, Strockbine NA, Panchalingam S, Tennant SM, Barry EM, Marohn ME, Antonio M, Hossain A, Mandomando I, Ochieng JB, Oundo JO, Qureshi S, Ramamurthy T, Tamboura B, Adegbola RA, Hossain MJ, Saha D, Sen S, Faruque AS, Alonso PL, Breiman RF, Zaidi AK, Sur D, Sow SO, Berkeley LY, O'Reilly CE, Mintz ED, Biswas K, Cohen D, Farag TH, Nasrin D, Wu Y, Blackwelder WC, Kotloff KL, Nataro JP, Levine MM. 2014. Shigella isolates from the global enteric multicenter study inform vaccine development. Clin Infect Dis 59:933-41.

3. Holt KE, Baker S, Weill FX, Holmes EC, Kitchen A, Yu J, Sangal V, Brown DJ, Coia JE, Kim DW, Choi SY, Kim SH, Da Silveira WD, Pickard DJ, Farrar JJ, Parkhill J, Dougan G, Thomson NR. 2012. Shigella sonnei genome sequencing and phylogenetic analysis indicate recent global dissemination from Europe. Nature Genetics 44:1056-1059.

4. Bangtrakulnonth A, Vieira AR, Lo DMA, Wong F, Pornreongwong S, Pulsrikarn C, Sawanpanyalert $P$, Hendriksen RS, Aarestrup FM. 2008. Shigella from Humans in Thailand During 1993 to 2006: Spatial-Time Trends in Species and Serotype Distribution. Foodborne Pathogens and Disease 5:773-784. 
5. Mao Y, Cui E, Bao C, Liu Z, Chen S, Zhang J, Wang H, Zhang C, Zou J, Klena JD, Zhu B, Qu F, Wang Z. 2013. Changing trends and serotype distribution of Shigella species in Beijing from 1994 to 2010. Gut pathogens 5:21.

6. Sousa MÂB, Mendes EN, Collares GB, Péret-Filho LA, Penna FJ, Magalhães PP. 2013. Shigella in Brazilian children with acute diarrhoea: prevalence, antimicrobial resistance and virulence genes. Memórias do Instituto Oswaldo Cruz 108:30-5.

7. Brotcke Zumsteg A, Goosmann C, Brinkmann V, Morona R, Zychlinsky A. 2014. IcsA Is a Shigella flexneri Adhesin Regulated by the Type III Secretion System and Required for Pathogenesis. Cell Host \& Microbe 15:435-445.

8. Mahmoud RY, Stones DH, Li W, Emara M, El-domany RA, Wang D, Wang Y, Krachler AM, Yu J. 2016. The Multivalent Adhesion Molecule SSO1327 plays a key role in Shigella sonnei pathogenesis. Molecular Microbiology 99:658-673.

9. Anderson MC, Vonaesch P, Saffarian A, Marteyn BS, Sansonetti PJ. 2017. Shigella sonnei Encodes a Functional T6SS Used for Interbacterial Competition and Niche Occupancy. Cell Host and Microbe 21:769-776.e3.

10. Caboni M, Pedron T, Rossi O, Goulding D, Pickard D, Citiulo F, MacLennan CA, Dougan G, Thomson NR, Saul A, Sansonetti PJ, Gerke C. 2015. An O antigen capsule modulates bacterial pathogenesis in Shigella sonnei. PLoS pathogens 11:e1004749.

11. Jiang Y, Yang F, Zhang X, Yang J, Chen L, Yan Y, Nie H, Xiong Z, Wang J, Dong J, Xue Y, Xu X, Zhu $Y$, Chen S, Jin Q. 2005. The complete sequence and analysis of the large virulence plasmid pSS of Shigella sonnei. Plasmid 54:149-59.

12. Liu B, Knirel YA, Feng L, Perepelov AV, Senchenkova SyN, Wang Q, Reeves PR, Wang L. 2008. Structure and genetics of $<i>$ Shigella</i $>0$ antigens. FEMS Microbiology Reviews 32:627-653.

13. Bergsbaken T, Fink SL, Cookson BT. 2009. Pyroptosis: host cell death and inflammation. Nature reviews Microbiology 7:99-109.

14. Mounier J, Vasselon T, Hellio R, Lesourd M, Sansonetti PJ. 1992. Shigella flexneri enters human colonic Caco-2 epithelial cells through the basolateral pole. Infection and immunity 60:23748.

15. Broz P, Dixit VM. 2016. Inflammasomes: mechanism of assembly, regulation and signalling. Nature Reviews Immunology 16:407-420.

16. Kayagaki N, Warming S, Lamkanfi M, Walle LV, Louie S, Dong J, Newton K, Qu Y, Liu J, Heldens S, Zhang J, Lee WP, Roose-Girma M, Dixit VM. 2011. Non-canonical inflammasome activation targets caspase-11. Nature 479:117-121.

17. Kayagaki N, Stowe IB, Lee BL, O'Rourke K, Anderson K, Warming S, Cuellar T, Haley B, RooseGirma M, Phung QT, Liu PS, Lill JR, Li H, Wu J, Kummerfeld S, Zhang J, Lee WP, Snipas SJ, Salvesen GS, Morris LX, Fitzgerald L, Zhang Y, Bertram EM, Goodnow CC, Dixit VM. 2015. Caspase-11 cleaves gasdermin $D$ for non-canonical inflammasome signalling. Nature 526:666671.

18. He W-t, Wan H, Hu L, Chen P, Wang X, Huang Z, Yang Z-H, Zhong C-Q, Han J. 2015. Gasdermin $D$ is an executor of pyroptosis and required for interleukin-1 $\beta$ secretion. Cell Research 25:1285-1298.

19. Shi J, Zhao Y, Wang K, Shi X, Wang Y, Huang H, Zhuang Y, Cai T, Wang F, Shao F. 2015. Cleavage of GSDMD by inflammatory caspases determines pyroptotic cell death. Nature 526:660-665.

20. Suzuki S, Mimuro H, Kim M, Ogawa M, Ashida H, Toyotome T, Franchi L, Suzuki M, Sanada T, Suzuki T, Tsutsui H, Núñez G, Sasakawa C. 2014. Shigella IpaH7.8 E3 ubiquitin ligase targets glomulin and activates inflammasomes to demolish macrophages. Proceedings of the National Academy of Sciences of the United States of America 111:E4254-63.

21. Suzuki S, Franchi L, He Y, Muñoz-Planillo R, Mimuro H, Suzuki T, Sasakawa C, Núñez G. 2014. Shigella Type III Secretion Protein Mxil Is Recognized by Naip2 to Induce NIrc4 Inflammasome Activation Independently of Pkcס. PLoS Pathogens 10:e1003926. 
22. Yang J, Zhao Y, Shi J, Shao F. 2013. Human NAIP and mouse NAIP1 recognize bacterial type III secretion needle protein for inflammasome activation. Proceedings of the National Academy of Sciences of the United States of America 110:14408-13.

23. Muñoz-Planillo R, Kuffa P, Martínez-Colón G, Smith BL, Rajendiran TM, Núñez G. 2013. K+ efflux is the common trigger of NLRP3 inflammasome activation by bacterial toxins and particulate matter. Immunity 38:1142-53.

24. Jorgensen I, Zhang Y, Krantz BA, Miao EA. 2016. Pyroptosis triggers pore-induced intracellular traps (PITs) that capture bacteria and lead to their clearance by efferocytosis. The Journal of Experimental Medicine 213:2113-2128.

25. Liu X, Zhang Z, Ruan J, Pan Y, Magupalli VG, Wu H, Lieberman J. 2016. Inflammasome-activated gasdermin D causes pyroptosis by forming membrane pores. Nature 535:153-158.

26. High N, Mounier J, Prévost MC, Sansonetti PJ. 1992. IpaB of Shigella flexneri causes entry into epithelial cells and escape from the phagocytic vacuole. The EMBO journal 11:1991-9.

27. Knodler LA, Nair V, Steele-Mortimer O. 2014. Quantitative Assessment of Cytosolic Salmonella in Epithelial Cells. PLoS ONE 9:e84681.

28. Mellouk N, Weiner A, Aulner N, Schmitt C, Elbaum M, Shorte SL, Danckaert A, Enninga J. 2014. Shigella subverts the host recycling compartment to rupture its vacuole. Cell Host Microbe 16:517-30.

29. Coll RC, Robertson AA, Chae JJ, Higgins SC, Munoz-Planillo R, Inserra MC, Vetter I, Dungan LS, Monks BG, Stutz A, Croker DE, Butler MS, Haneklaus M, Sutton CE, Nunez G, Latz E, Kastner DL, Mills KH, Masters SL, Schroder K, Cooper MA, O'Neill LA. 2015. A small-molecule inhibitor of the NLRP3 inflammasome for the treatment of inflammatory diseases. Nat Med 21:248-55.

30. McVicker G, Tang CM. 2016. Deletion of toxin-antitoxin systems in the evolution of Shigella sonnei as a host-adapted pathogen. Nat Microbiol 2:16204.

31. Long ME, Lindemann SR, Rasmussen JA, Jones BD, Allen LA. 2013. Disruption of Francisella tularensis Schu S4 igll, iglJ, and pdpC genes results in attenuation for growth in human macrophages and in vivo virulence in mice and reveals a unique phenotype for $\mathrm{pdpC}$. Infect Immun 81:850-61.

32. Brodmann M, Dreier RF, Broz P, Basler M. 2017. Francisella requires dynamic type VI secretion system and ClpB to deliver effectors for phagosomal escape. Nat Commun 8:15853.

33. Xu D-Q, Cisar JO, Ambulos N, Burr DH, Kopecko DJ. 2002. Molecular cloning and characterization of genes for Shigella sonnei form I O polysaccharide: proposed biosynthetic pathway and stable expression in a live salmonella vaccine vector. Infection and immunity 70:4414-23.

34. Allison GE, Verma NK. 2000. Serotype-converting bacteriophages and O-antigen modification in Shigella flexneri. Trends in Microbiology 8:17-23.

35. Dharmasena MN, Hanisch BW, Wai TT, Kopecko DJ. 2013. Stable expression of Shigella sonnei form I O-polysaccharide genes recombineered into the chromosome of live Salmonella oral vaccine vector Ty21a. International Journal of Medical Microbiology 303:105-113.

36. Dharmasena MN, Osorio M, Takeda K, Stibitz S, Kopecko DJ. 2017. Stable chromosomal expression of shigella flexneri $2 \mathrm{a}$ and $3 \mathrm{a}$ o-antigens in the live salmonella oral vaccine vector Ty21a. Clinical and Vaccine Immunology 24.

37. Schroeder GN, Hilbi H. 2008. Molecular pathogenesis of Shigella spp.: controlling host cell signaling, invasion, and death by type III secretion. Clin Microbiol Rev 21:134-56.

38. Case CL, Shin S, Roy CR. 2009. Asc and Ipaf Inflammasomes Direct Distinct Pathways for Caspase-1 Activation in Response to Legionella pneumophila. Infection and Immunity 77:1981-1991.

39. Mariathasan S, Newton K, Monack DM, Vucic D, French DM, Lee WP, Roose-Girma M, Erickson S, Dixit VM. 2004. Differential activation of the inflammasome by caspase- 1 adaptors ASC and Ipaf. Nature 430:213-218. 
40. Suzuki T, Franchi L, Toma C, Ashida H, Ogawa M, Yoshikawa Y, Mimuro H, Inohara N, Sasakawa C, Nuñez G. 2007. Differential Regulation of Caspase-1 Activation, Pyroptosis, and Autophagy via Ipaf and ASC in Shigella-Infected Macrophages. PLoS Pathogens 3:e111.

41. Reeves P. 1995. Role of O-antigen variation in the immune response. Trends Microbiol 3:3816.

42. Duerr CU, Zenk SF, Chassin C, Pott J, Gutle D, Hensel M, Hornef MW. 2009. O-antigen delays lipopolysaccharide recognition and impairs antibacterial host defense in murine intestinal epithelial cells. PLoS Pathog 5:e1000567.

43. Saldías MS, Ortega X, Valvano MA. 2009. Burkholderia cenocepacia $O$ antigen lipopolysaccharide prevents phagocytosis by macrophages and adhesion to epithelial cells. Journal of Medical Microbiology 58:1542-1548.

44. Murray GL, Attridge SR, Morona R. 2006. Altering the Length of the Lipopolysaccharide $O$ Antigen Has an Impact on the Interaction of Salmonella enterica Serovar Typhimurium with Macrophages and Complement. JOURNAL OF BACTERIOLOGY 188:2735-2739.

45. West NP, Sansonetti P, Mounier J, Exley RM, Parsot C, Guadagnini S, Prevost MC, ProchnickaChalufour A, Delepierre M, Tanguy M, Tang CM. 2005. Optimization of virulence functions through glucosylation of Shigella LPS. Science 307:1313-7.

46. Goddard PJ, Sanchez-Garrido J, Slater SL, Kalyan M, Ruano-Gallego D, Marches O, Fernandez LA, Frankel G, Shenoy AR. 2019. Enteropathogenic Escherichia coli Stimulates Effector-Driven Rapid Caspase-4 Activation in Human Macrophages. Cell Rep 27:1008-1017 e6.

47. Fisch D, Bando H, Clough B, Hornung V, Yamamoto M, Shenoy AR, Frickel EM. 2019. Human GBP1 is a microbe-specific gatekeeper of macrophage apoptosis and pyroptosis. EMBO J doi:10.15252/embj.2018100926.

48. Reyes Ruiz VM, Ramirez J, Naseer N, Palacio NM, Siddarthan IJ, Yan BM, Boyer MA, Pensinger DA, Sauer JD, Shin S. 2017. Broad detection of bacterial type III secretion system and flagellin proteins by the human NAIP/NLRC4 inflammasome. Proc Natl Acad Sci U S A 114:1324213247.

49. Kortmann J, Brubaker SW, Monack DM. 2015. Cutting Edge: Inflammasome Activation in Primary Human Macrophages Is Dependent on Flagellin. J Immunol 195:815-9.

50. Eldridge MJG, Sanchez-Garrido J, Hoben GF, Goddard PJ, Shenoy AR. 2017. The Atypical Ubiquitin E2 Conjugase UBE2L3 Is an Indirect Caspase-1 Target and Controls IL-1 $\beta$ Secretion by Inflammasomes. Cell reports 18:1285-1297.

51. Schmid-Burgk JL, Gaidt MM, Schmidt T, Ebert TS, Bartok E, Hornung V. 2015. Caspase-4 mediates non-canonical activation of the NLRP3 inflammasome in human myeloid cells. European Journal of Immunology 45:2911-2917.

52. Eldridge MJG, Sanchez-Garrido J, Hoben GF, Goddard PJ, Shenoy AR. 2017. The Atypical Ubiquitin E2 Conjugase UBE2L3 Is an Indirect Caspase-1 Target and Controls IL-1beta Secretion by Inflammasomes. Cell Rep 18:1285-1297.

53. Tsai CM, Frasch CE. 1982. A sensitive silver stain for detecting lipopolysaccharides in polyacrylamide gels. Anal Biochem 119:115-9.

54. Formal SB, Kent TH, May HC, Palmer A, Falkow S, LaBrec EH. 1966. Protection of monkeys against experimental shigellosis with a living attenuated oral polyvalent dysentery vaccine. Journal of bacteriology 92:17-22.

55. Watson J, Jenkins C, Clements A. 2018. Shigella sonnei Does Not Use Amoebae as Protective Hosts. Applied and environmental microbiology 84:e02679-17.

56. Sansonetti PJ, Kopecko DJ, Formal1 ASB. 1982. Involvement of a Plasmid in the Invasive Ability of Shigella flexneri. INFECTION AND IMMUNITY 35:852-860.

57. Formal SB, Dammin GJ, Labrec EH, Schneider H. 1958. Experimental Shigella infections: characteristics of a fatal infection produced in guinea pigs. Journal of bacteriology 75:604-610. 
58. Figurski DH, Helinski DR. 1979. Replication of an origin-containing derivative of plasmid RK2 dependent on a plasmid function provided in trans. Proceedings of the National Academy of Sciences of the United States of America 76:1648-52.

59. Herrero M, de Lorenzo V, Timmis KN. 1990. Transposon vectors containing non-antibiotic resistance selection markers for cloning and stable chromosomal insertion of foreign genes in gram-negative bacteria. Journal of bacteriology 172:6557-67.

60. Herring CD, Glasner JD, Blattner FR. 2003. Gene replacement without selection: regulated suppression of amber mutations in Escherichia coli. Gene 311:153-63. 
Strain

Details

Source

\begin{tabular}{|c|c|c|}
\hline S. sonnei $53 \mathrm{G}$ & & (54) \\
\hline S. sonnei 381 & Clinical isolate $\mathrm{H} 140860381$ & $(55)$ \\
\hline S. flexneri M90T & Serotype $5 a$ & $(56)$ \\
\hline S. flexneri $2457 \mathrm{~T}$ & Serotype $2 a$ & $(57)$ \\
\hline S. sonnei $\Delta \mathrm{T} 3 \mathrm{SS}$ & $\begin{array}{l}m x i D \text { replaced with aphA-3, conferring kanamycin } \\
\text { resistance }\end{array}$ & $(55)$ \\
\hline S. flexneri $\Delta$ T3SS & $\begin{array}{l}m x i D \text { replaced with aphA-3, conferring } \\
\text { kanamycin resistance }\end{array}$ & $(55)$ \\
\hline S. sonnei $\Delta \mathbf{G} 4 \mathrm{C}$ & $\begin{array}{l}g 4 c \text { cluster (genes } y m c D C B A, y c c Z \text {, etp, etk) } \\
\text { replaced with erm cassette conferring resistance } \\
\text { to erythromycin }\end{array}$ & $\begin{array}{l}\text { A kind gift from } \mathrm{C} \text {. } \\
\text { Gerke (10) }\end{array}$ \\
\hline E. coli helper & $\begin{array}{l}\text { E. coli } 1047 \text { harbouring pRK2013 which encodes } \\
\text { RK2 transfer genes }\end{array}$ & $(58)$ \\
\hline E. coli CC118-גpir & Expresses $\lambda$ protein from a lysogenic phage & (59) \\
\hline $\begin{array}{l}\text { S. sonnei } 53 \mathrm{G} \\
\text { receiver }\end{array}$ & $\begin{array}{l}\text { S. sonnei } 53 G \text { harbouring pACBSR which } \\
\text { encodes arabinose inducible lamba red } \\
\text { recombinase and I-Scel endonuclease }\end{array}$ & $(60)$ \\
\hline $\begin{array}{l}\text { S. sonnei } \\
\text { LVP }\end{array}$ & $\begin{array}{l}\text { S. sonnei 53G with cat (conferring } \\
\text { chloramphenicol resistance) inserted at nt } 83716 \\
\text { of } 53 G \text { LVP, allowing selection of LVP positive } \\
\text { strains }\end{array}$ & \\
\hline S. sonnei $\Delta$ waaL & $\begin{array}{l}\text { S. sonnei } 53 G \text { with waaL replaced with aphA-3, } \\
\text { conferring kanamycin resistance }\end{array}$ & This study \\
\hline S. sonnei $\Delta \mathrm{O}-\mathrm{Ag}$ & $\begin{array}{l}\text { S. sonnei 53G with O-Ag biosynthesis operon } \\
\text { (wbgTU, wzx, wzy, IS630, wbgVWXYZ) replaced } \\
\text { with aphA-3, conferring kanamycin resistance }\end{array}$ & This study \\
\hline S. sonnei $\Delta t s s B$ & $\begin{array}{l}\text { S. sonnei } 53 G \text { with } t s s V \text { replaced with aphA-3, } \\
\text { conferring kanamycin resistance }\end{array}$ & This study \\
\hline $\begin{array}{l}\text { S. sonnei } \Delta \mathrm{O}-\mathrm{Ag} \\
+\mathrm{pO}-\mathrm{Ag}^{\text {ss }}\end{array}$ & $\begin{array}{l}\text { O-Ag biosynthesis operon (wzZ, wbgTU, wzX, } \\
\text { wzy, IS630, wbgVWXYZ with } 102 \text { bp } 5 \text { ' of wzz } \\
\text { and } 76 \text { bp } 3 \text { ' of wbgZ) from } 53 G \text { inserted into } \\
\text { pSEVA471:SmR expressed in a S. sonnei } 53 G \\
\triangle O A g \text { background }\end{array}$ & This study \\
\hline $\begin{array}{l}\text { S. sonnei 53G } \Delta O- \\
\mathrm{Ag}+\mathrm{pO}-\mathrm{Ag}^{\text {sf5a }}\end{array}$ & $\begin{array}{l}\text { gtr modification (gtrABV with } 100 \mathrm{bp} 5 \text { ' of } g t r A \text { and } \\
100 \text { bp } 3 \text { ' of gtrV) and O-Ag biosynthesis } \\
\text { (rfbBDACEFG, rfc, rfblJ, orf, orf with } 500 \mathrm{bp} 5 \text { ' of }\end{array}$ & This study \\
\hline
\end{tabular}


Table S2: Primers used in this study.

\begin{tabular}{|c|c|c|}
\hline No. & Name & Sequence (Restriction enzyme sites are underlined) \\
\hline 1 & 53G.nt82936.F & ATGTCGGAACAACGGAAG \\
\hline 2 & 53G.nt83715.p2.R & $\begin{array}{l}\text { CTAAGGAGGATATTCATATGCTTACAAAGCCTCA } \\
\text { TTAG }\end{array}$ \\
\hline 3 & 53G.nt83716.p1.F & $\begin{array}{l}\text { GCAGCTCCAGCCTACACAATTGTCGTTTTTTATC } \\
\text { TGTG }\end{array}$ \\
\hline 4 & 53G.nt84215.R & CTGTTTTATTCTCСTAАTCTTC \\
\hline 5 & 53G.nt84574.R & GCTATCTGTTATTCAATCC \\
\hline 6 & 53G.492bpustssB.F & CTGGATAACCATACCCAAC \\
\hline 7 & 53G.tssB.K.R & $\begin{array}{l}\text { GCAGCTCCAGCCTACACAGCTGCCTTCAAATTTT } \\
\text { TTGC }\end{array}$ \\
\hline 8 & 53G.tssB.K.F & $\begin{array}{l}\text { CTAAGGAGGATATTCATATGCCACCACTATTTCA } \\
\text { TTAAAC }\end{array}$ \\
\hline 9 & 53G.422bpdstssB.R & CATGCTGTCCACGGCCAG \\
\hline 10 & 53G.1786bpdstssB.F & GTTACTCCСTGTCTAACC \\
\hline 11 & 53G.500bpuswaaL.BamHI.F & ACGGATCCAAGATTTACGACTAGGAGAAG \\
\hline 12 & 53G.500bpuswaaL.p1.R & $\begin{array}{l}\text { GAAGCAGCTCCAGCCTACACAATAGTTTCAACC } \\
\text { TATGCTAC }\end{array}$ \\
\hline 13 & 53G.500bpdswaaL.p2.F & $\begin{array}{l}\text { CTAAGGAGGATATTCATATGTGAATATGTGAAAT } \\
\text { AAAATCAGC }\end{array}$ \\
\hline 14 & 53G.500bpdswaaL.EcoRI.R & GCTGAATTCCCGTATTTCTTCATGCGAC \\
\hline 15 & 53G.waaC.F & GAGCAATGATTACCCTTTAG \\
\hline 16 & $\begin{array}{l}\text { 53G.500bpuswbgT.BamH1. } \\
\text { F }\end{array}$ & CTAGGATCCAGTCTTGATGAGTTTGGTC \\
\hline 17 & 53G.500bpuswbgT.p1.R & $\begin{array}{l}\text { GAAGCAGCTCCAGCCTACACACTATTTCGCATT } \\
\text { GACACAAC }\end{array}$ \\
\hline 18 & 53G.500bpdswbgZ.p2.F & $\begin{array}{l}\text { CTAAGGAGGATATTCATATGTTGCTTAGGTTTAA } \\
\text { CCTTCC }\end{array}$ \\
\hline 19 & 53G.500bpdswbgZ.EcoRI.R & CTAGAATTCGTGAATGGAGGCCATAAC \\
\hline 20 & 53G.wZz.F & GCACCACGTATTGCTGAATTAG \\
\hline 21 & P1 & TGTGTAGGCTGGAGCTGCTTC \\
\hline 22 & P2 & CATATGAATATCCTCCTTAG \\
\hline 23 & 53G OAg.BamHI.F & CTAGGATCCCAGCGCTTTGGGAGCTGAAAC \\
\hline 24 & 53G OAg.BamHI.R & TAGGGATCCGGCGGTCACGATGTACCG \\
\hline 25 & M90T gtroperon.KpnI.F & $\begin{array}{l}\text { CTAGGTACCCTTATGTGTCTCAGTTTTGTCTCAT } \\
\text { CAG }\end{array}$ \\
\hline 26 & M90T gtroperon.BamHI.R & $\begin{array}{l}\text { GTGGGATCCAGAGGCCGTTAATTATAACTGCAG } \\
\text { ATG }\end{array}$ \\
\hline 27 & M90T OAg.BamHI.F & CTAGGATCCTGACCGGCGACAGTTACG \\
\hline
\end{tabular}




\begin{tabular}{lll}
\hline 28 & M90T OAg.Xbal.R & $\begin{array}{l}\text { AGATCTAGACAGAGATCCAAAAACTACGGACAT } \\
\text { AC }\end{array}$ \\
\hline 29 & M90T wzzB.Kpnl.F & CTAGGTACCCAAGCCGACGTCATTATCTC \\
\hline 30 & M90T wzzB.Kpnl.R & CTAGGTACCCAACACACGTAGGCCGGATAAG \\
\hline
\end{tabular}


A.

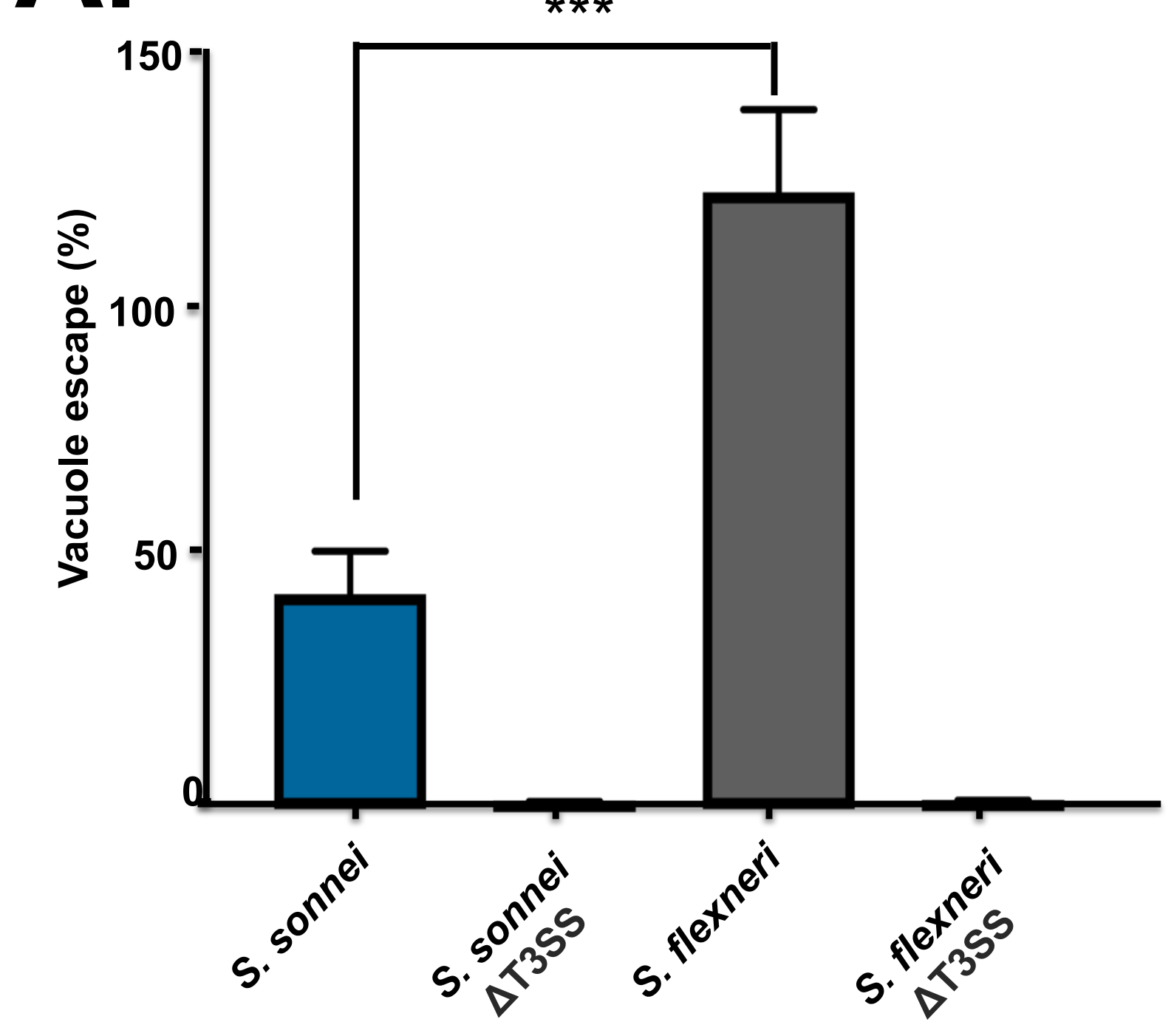

c.

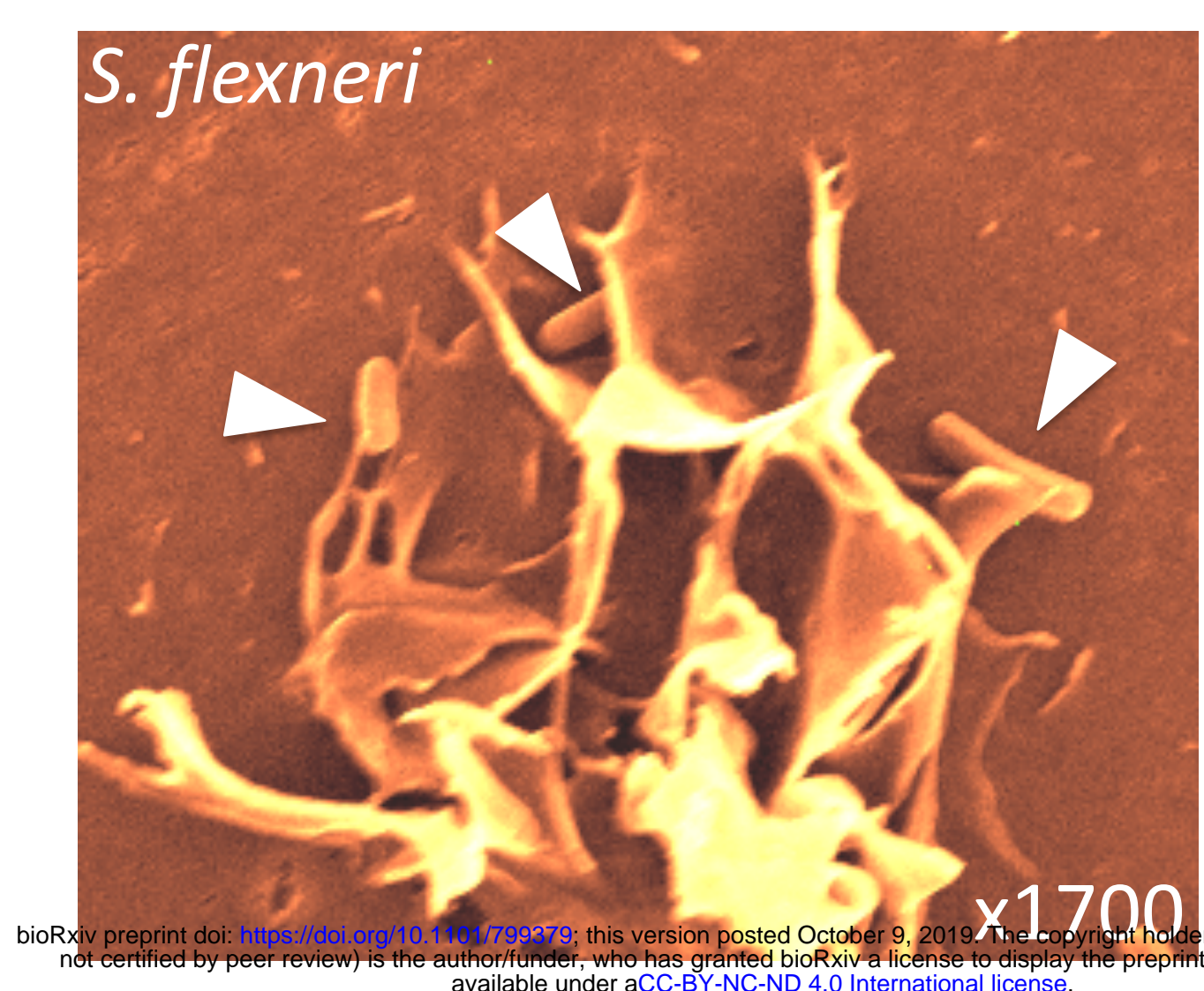

E.

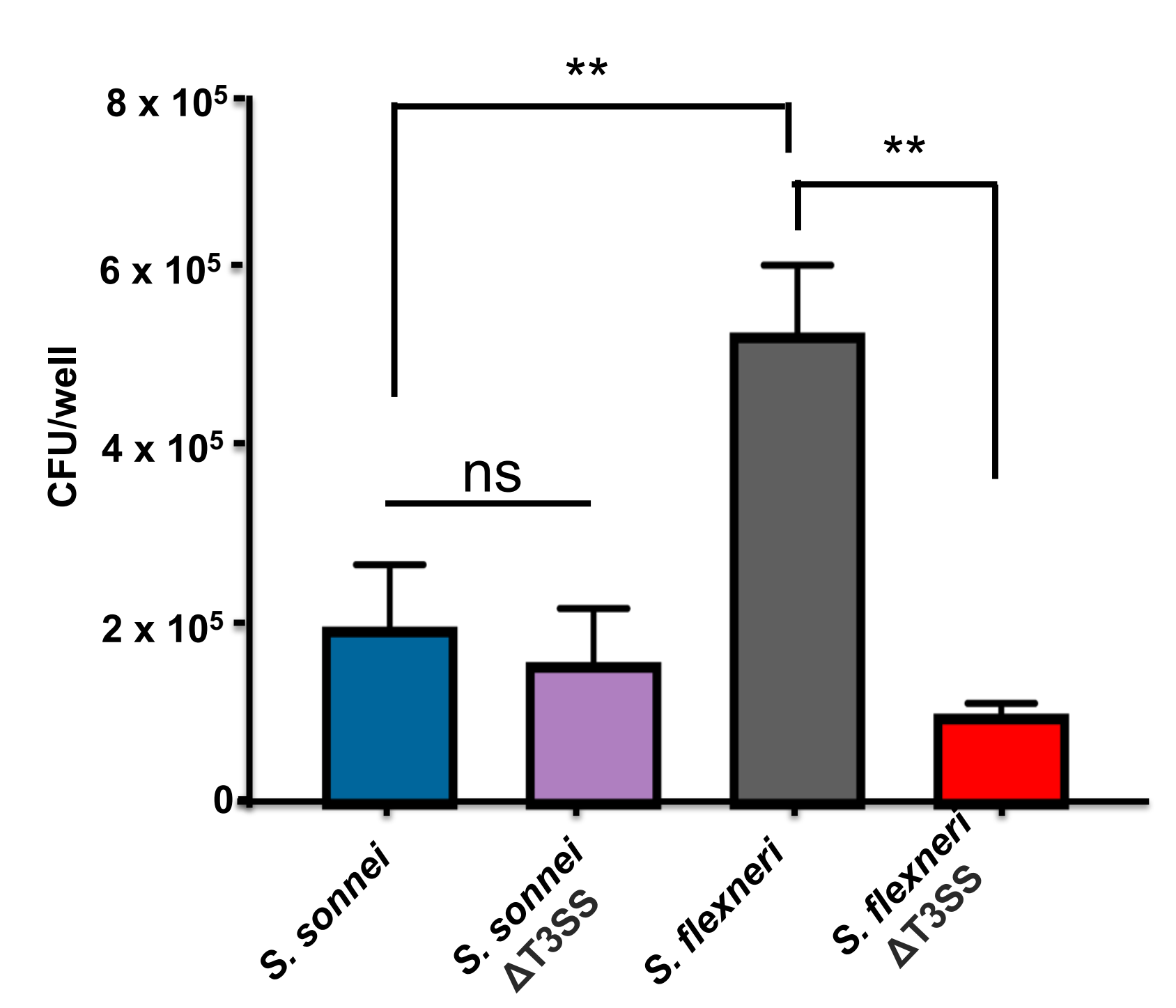

B.

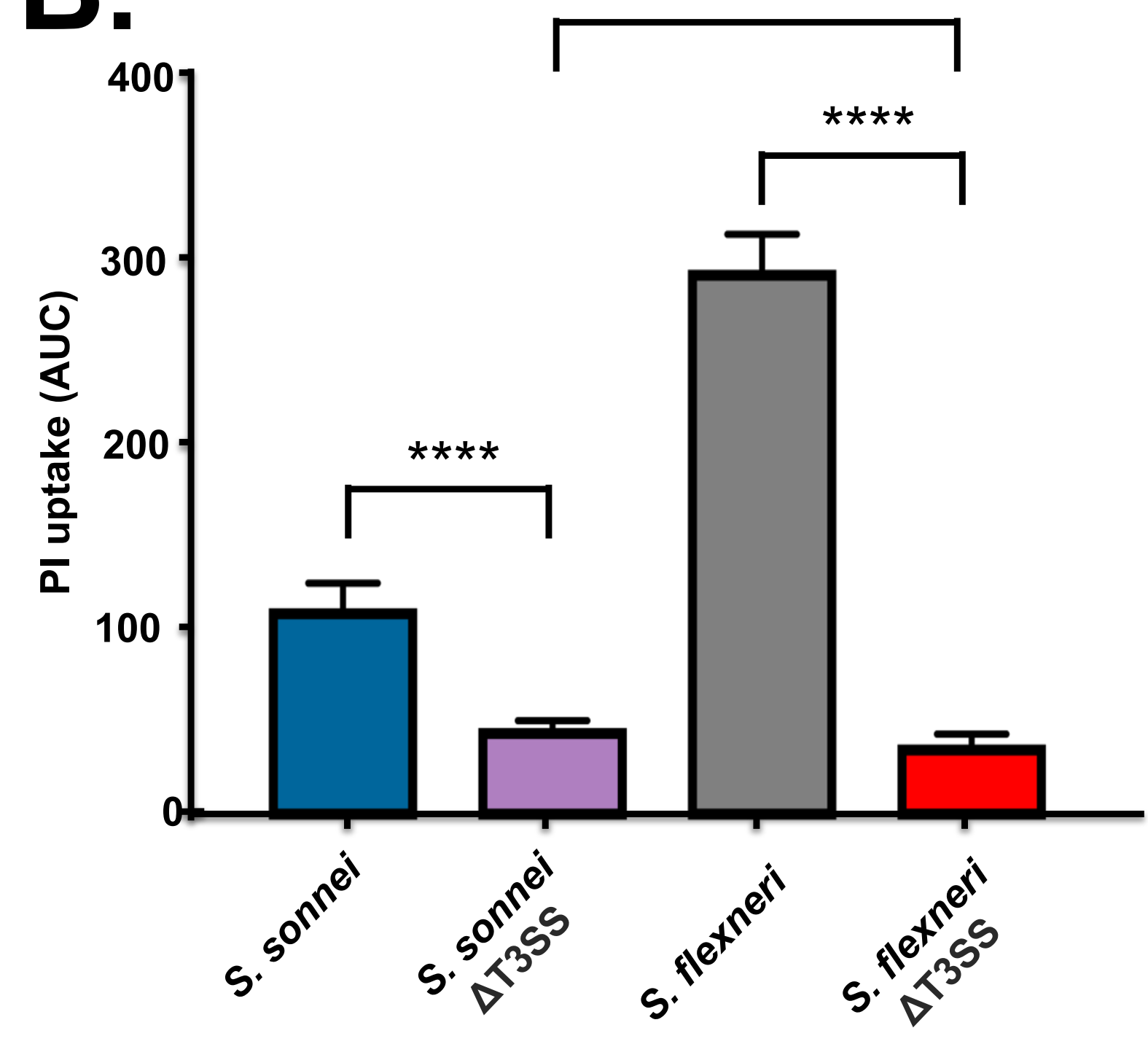

D.

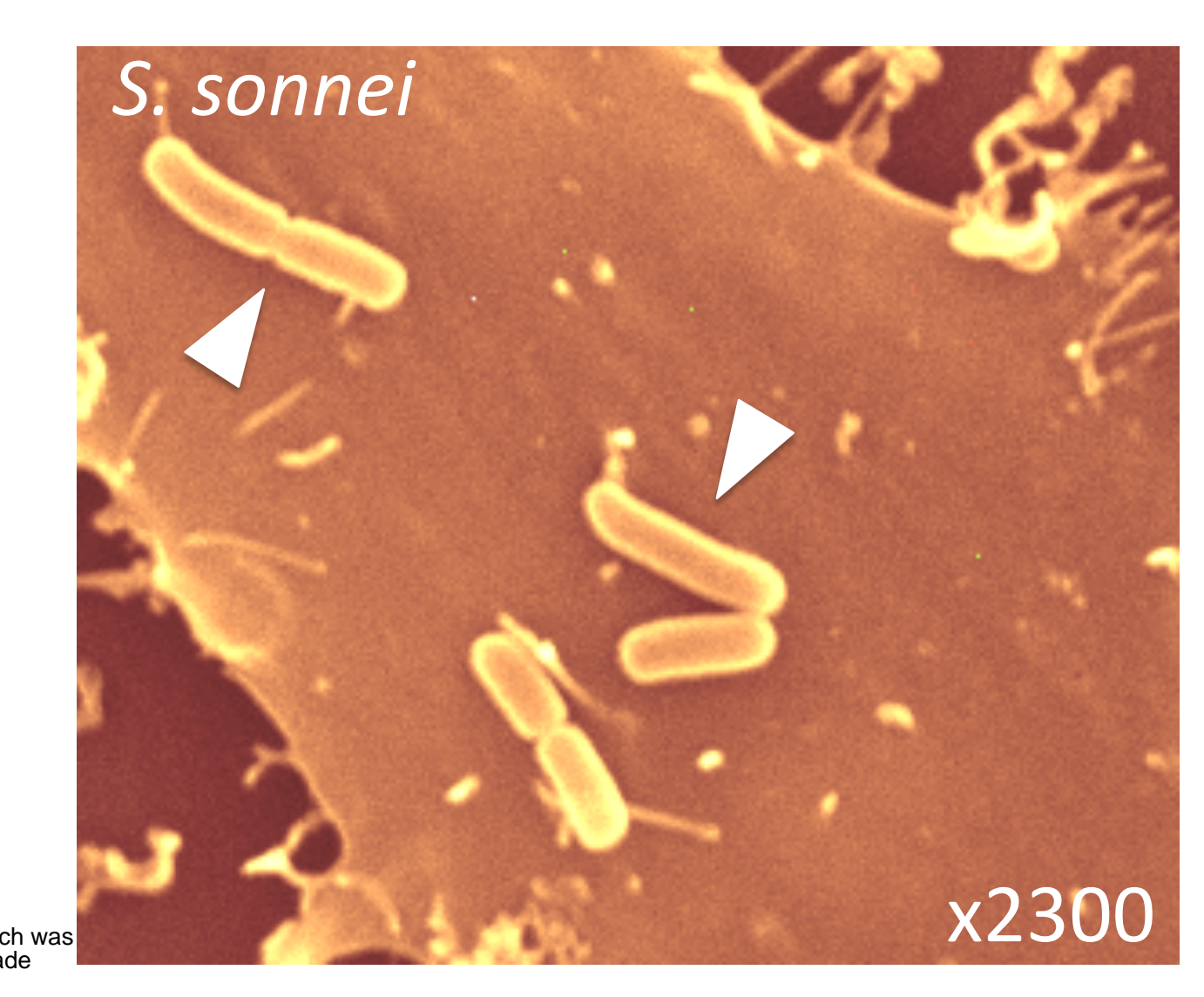

F.

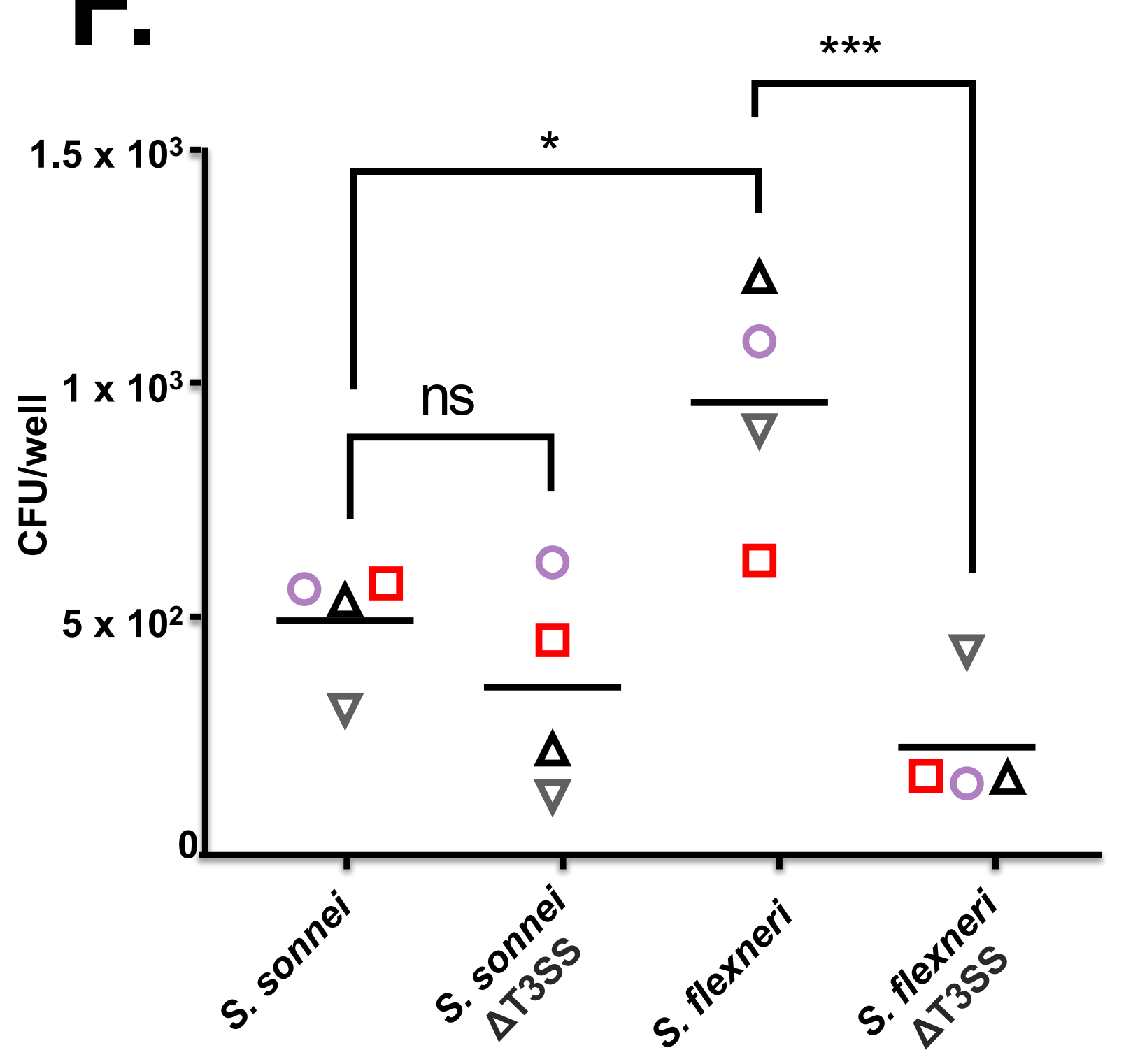

Figure 2 
A.

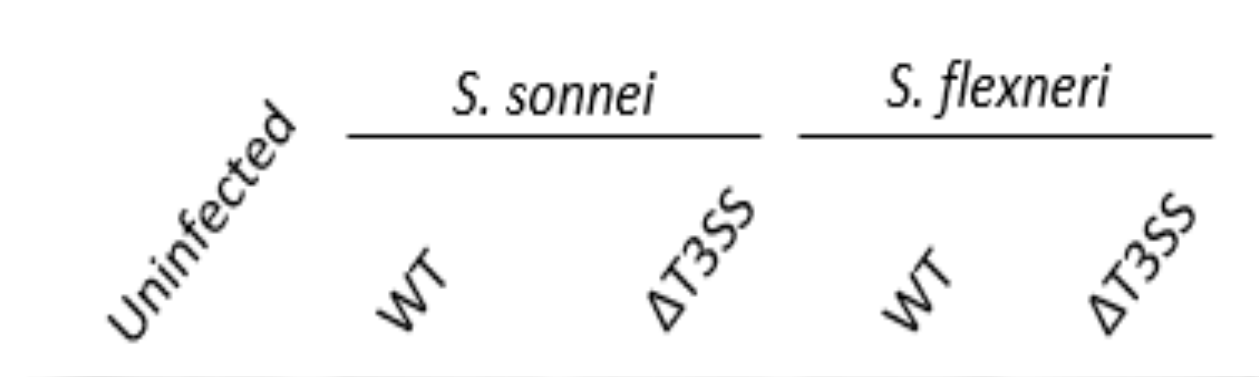

Caspase-1
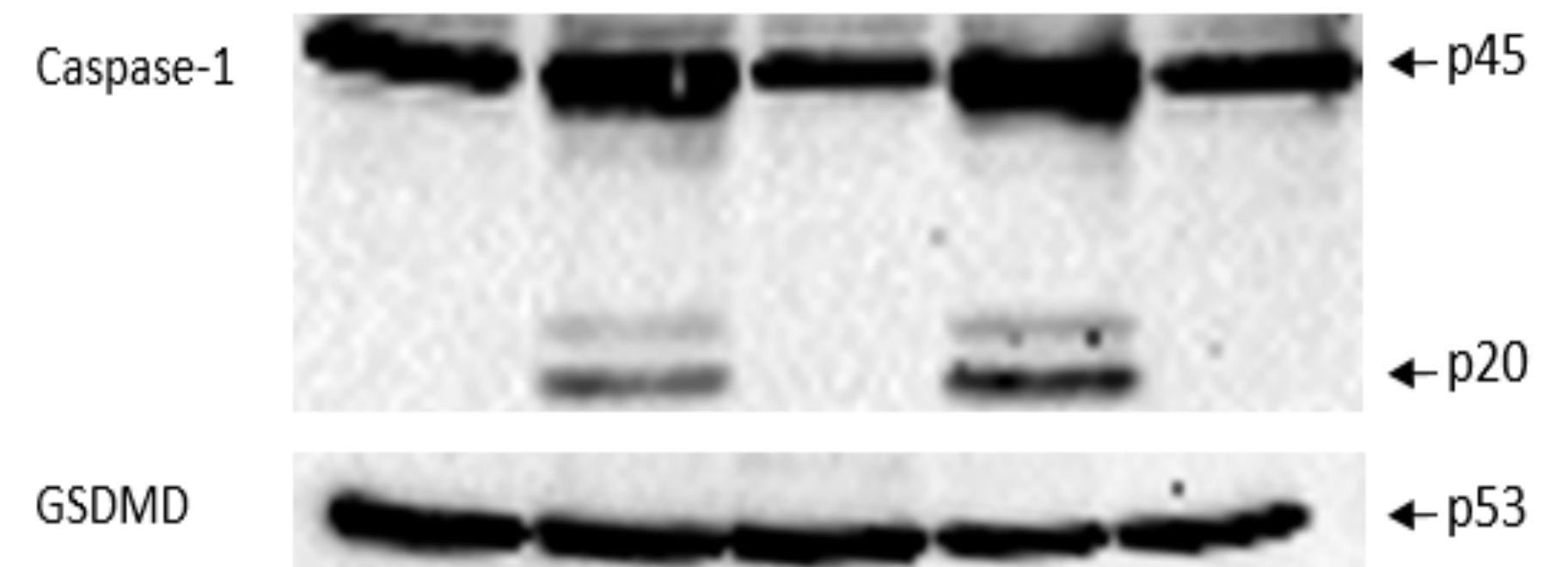

IL-18

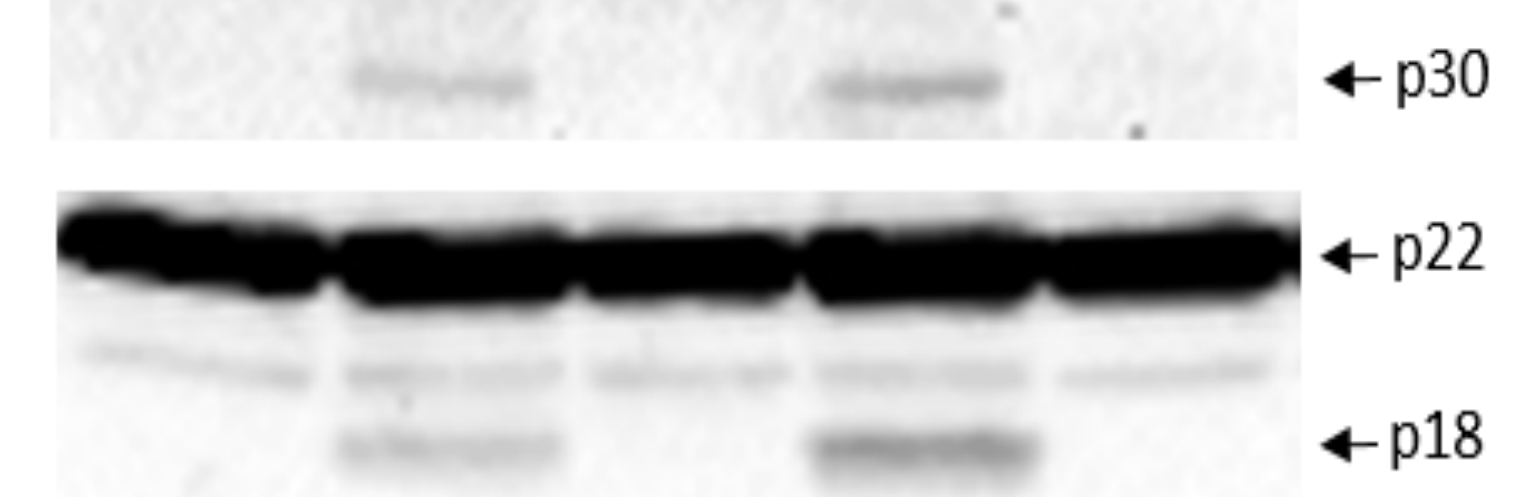

Actin

C.

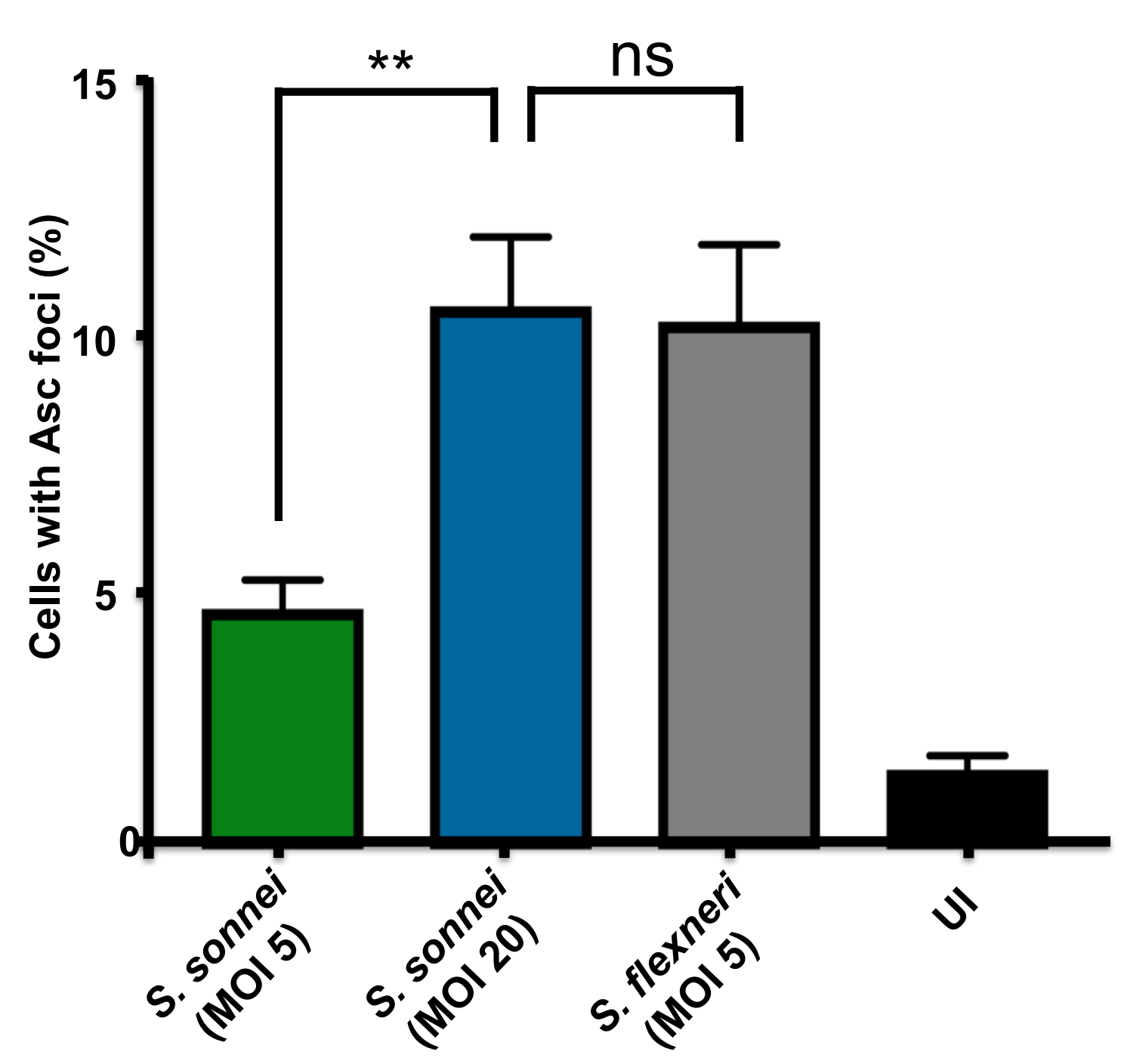

F.

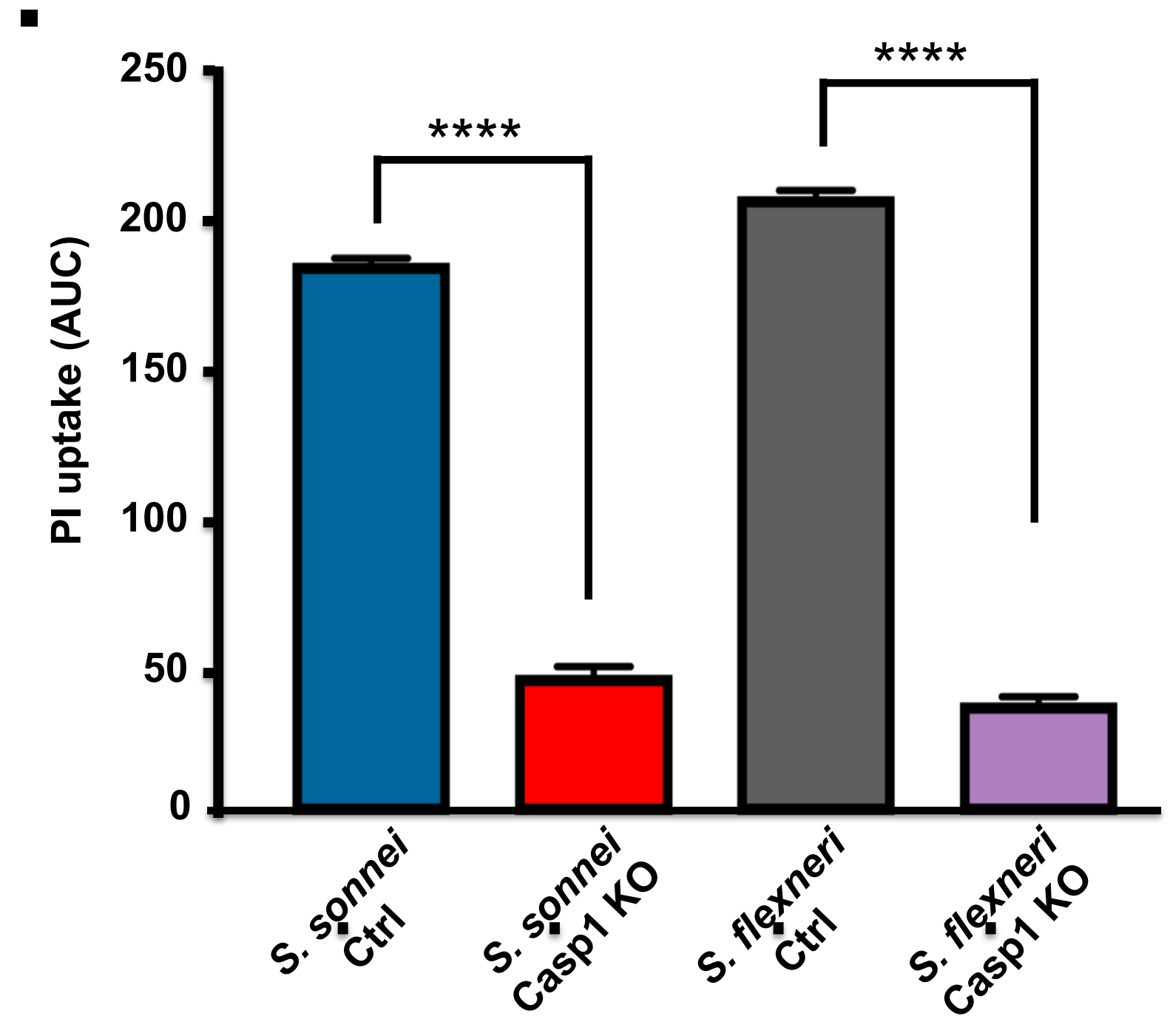

DAPI

Bacteria

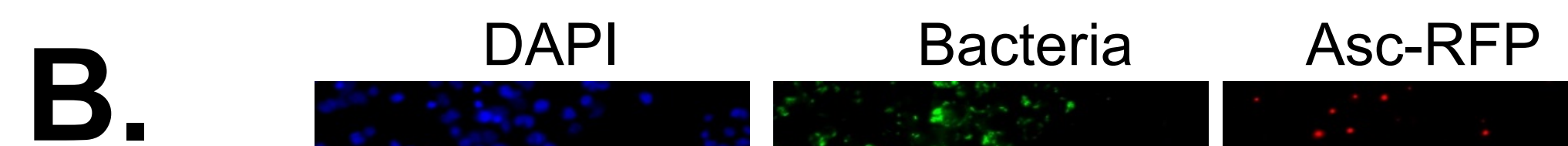

S. flexneri (MOI 5)

(MOI 5)

S. sonnei

(MOI 20)

Uninfected
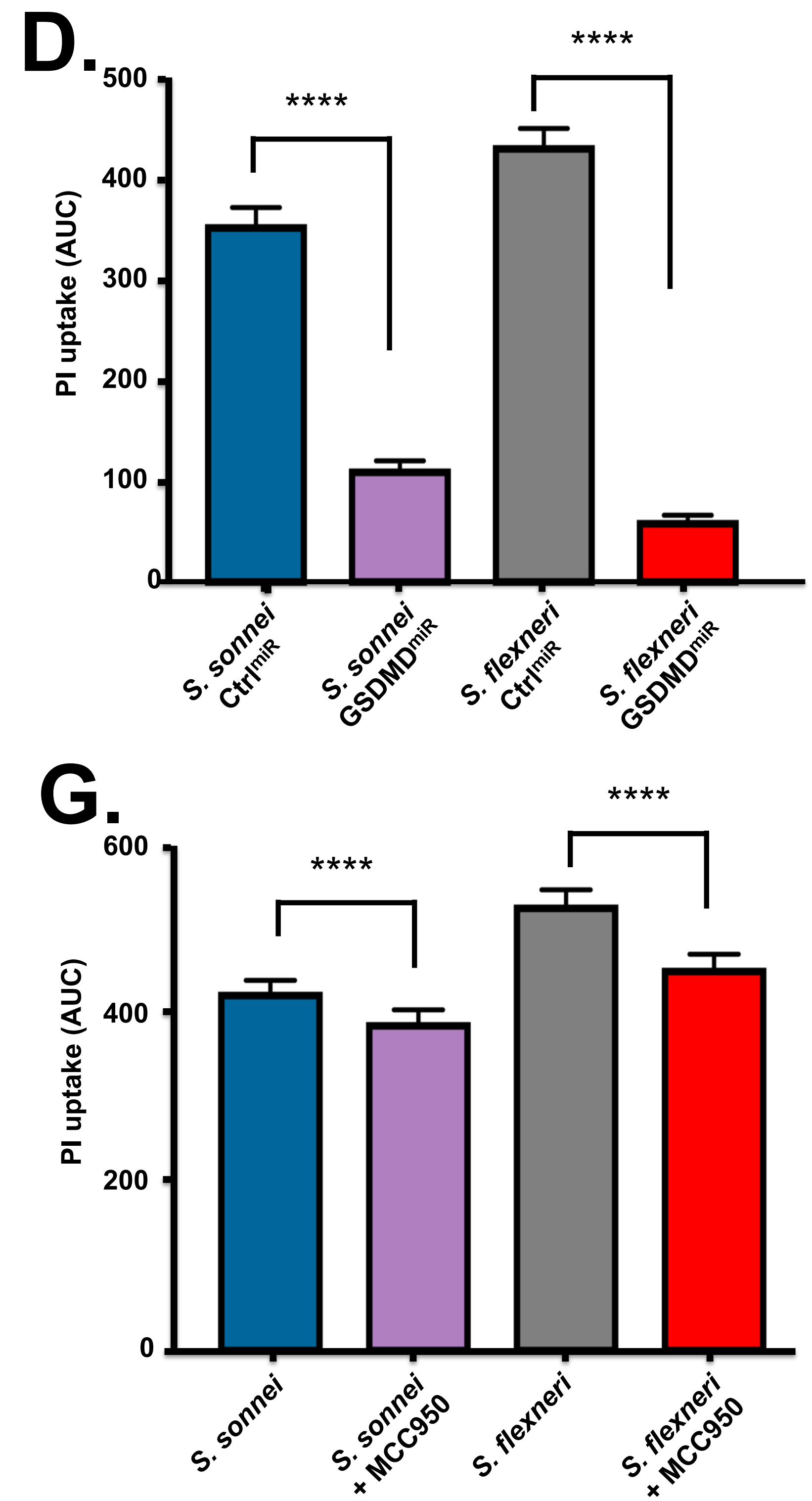

Asc-RFP

Merge
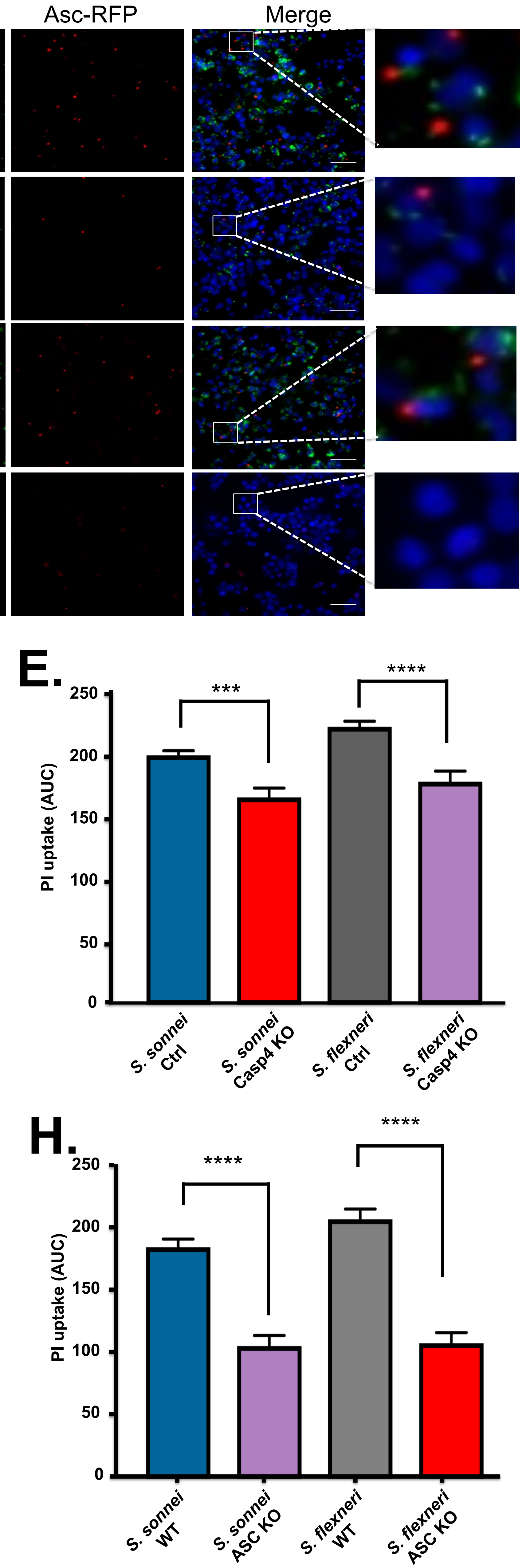

Figure 3 


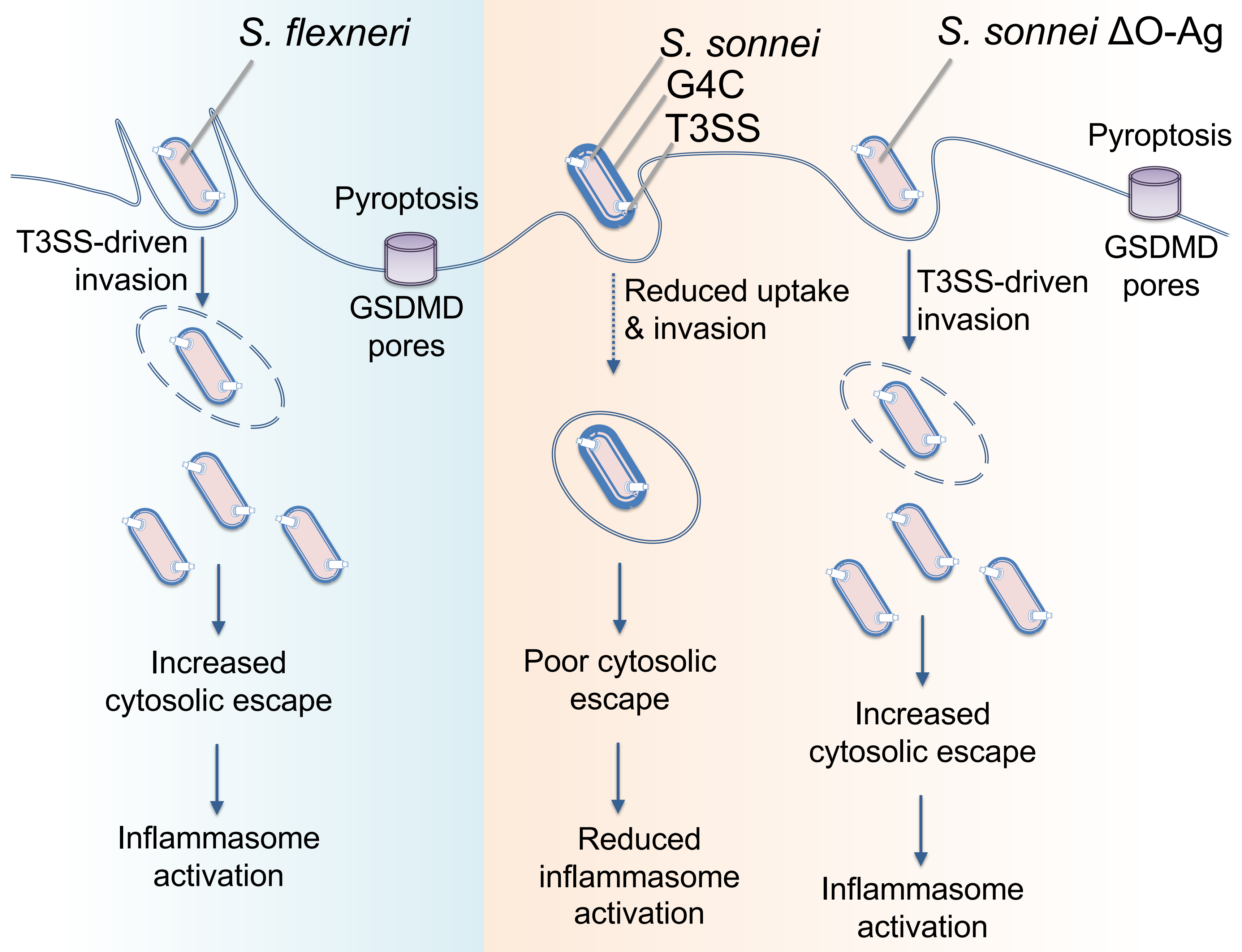

Figure 5 
A.

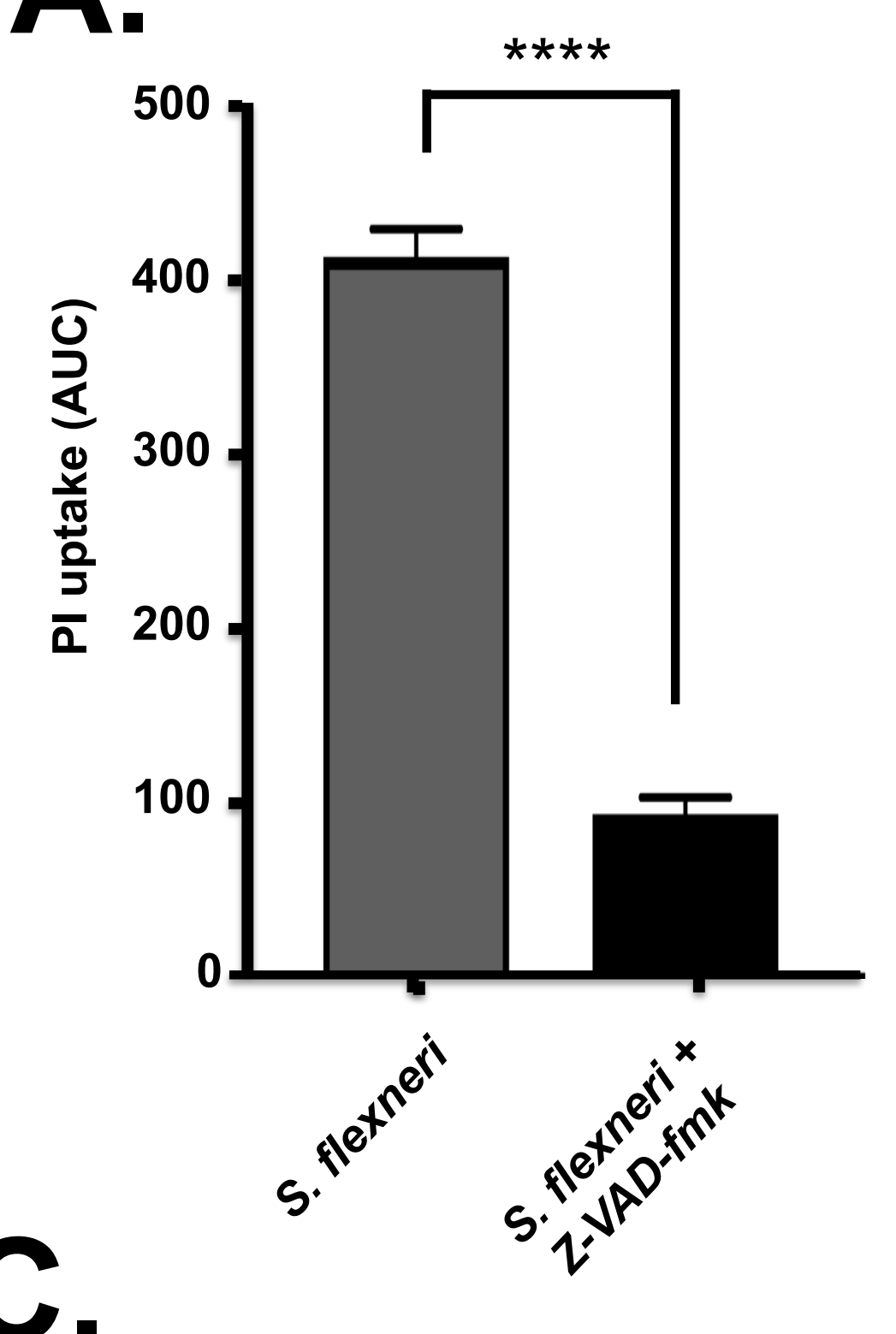

c.

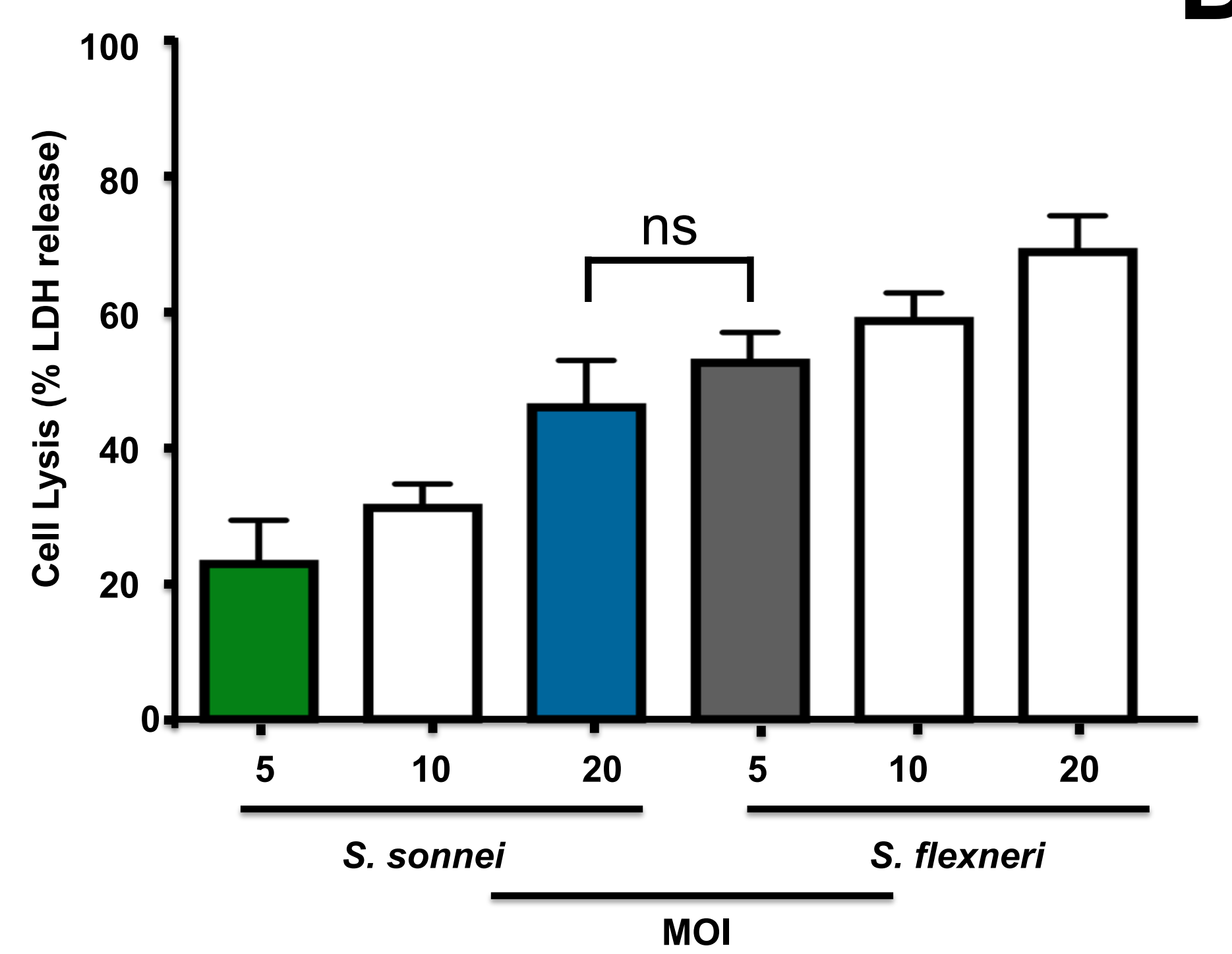

B. $1 \times 10^{\circ}$

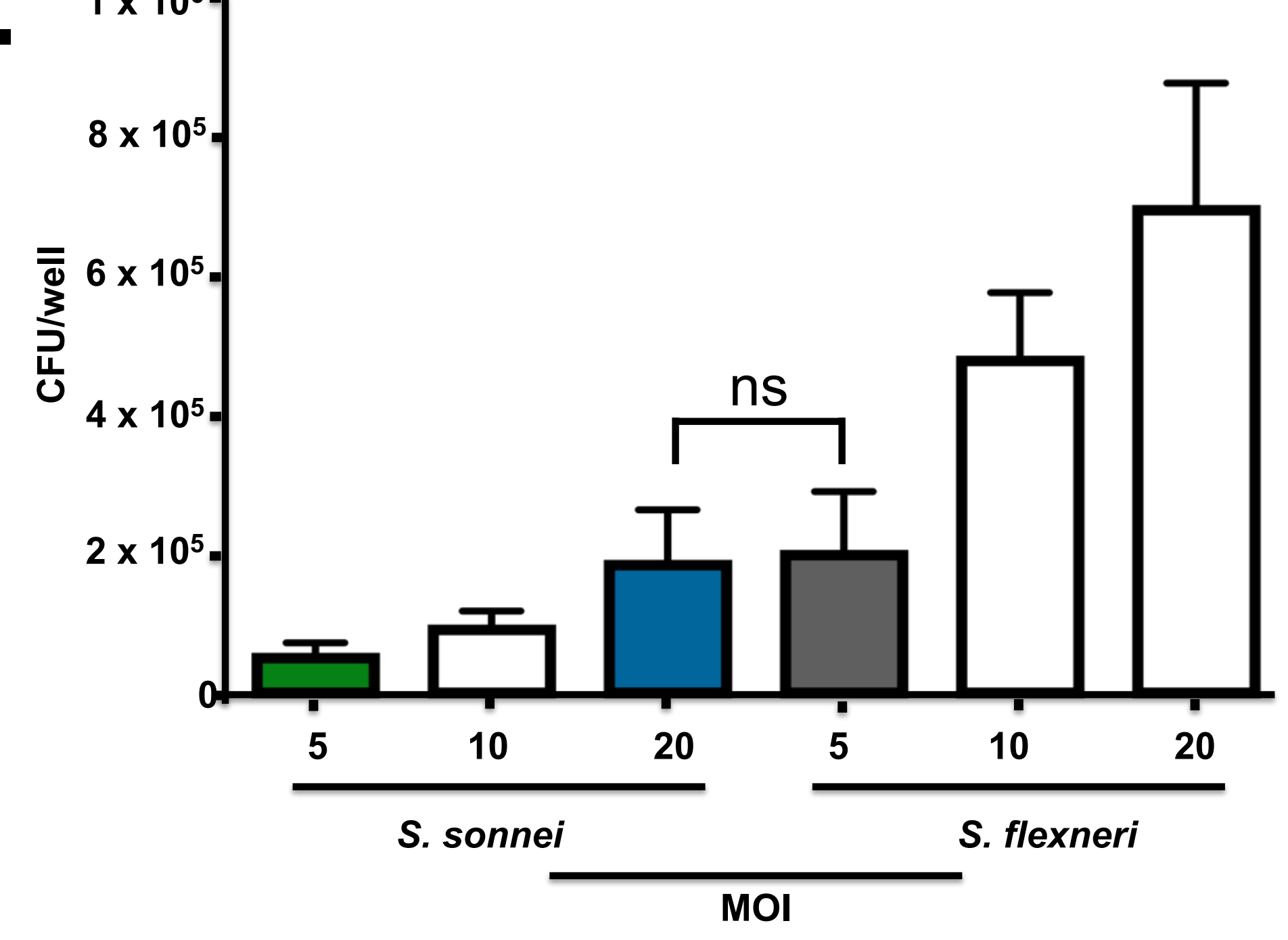

D.

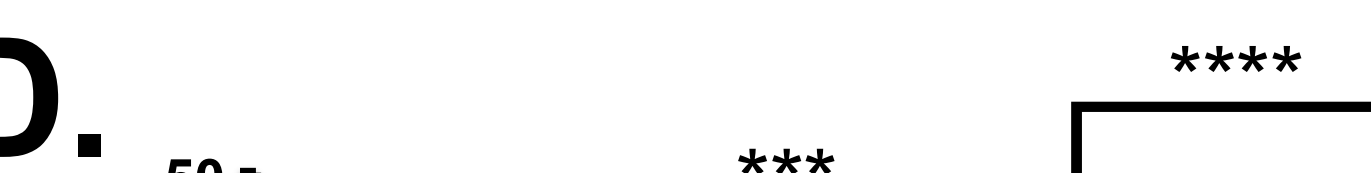




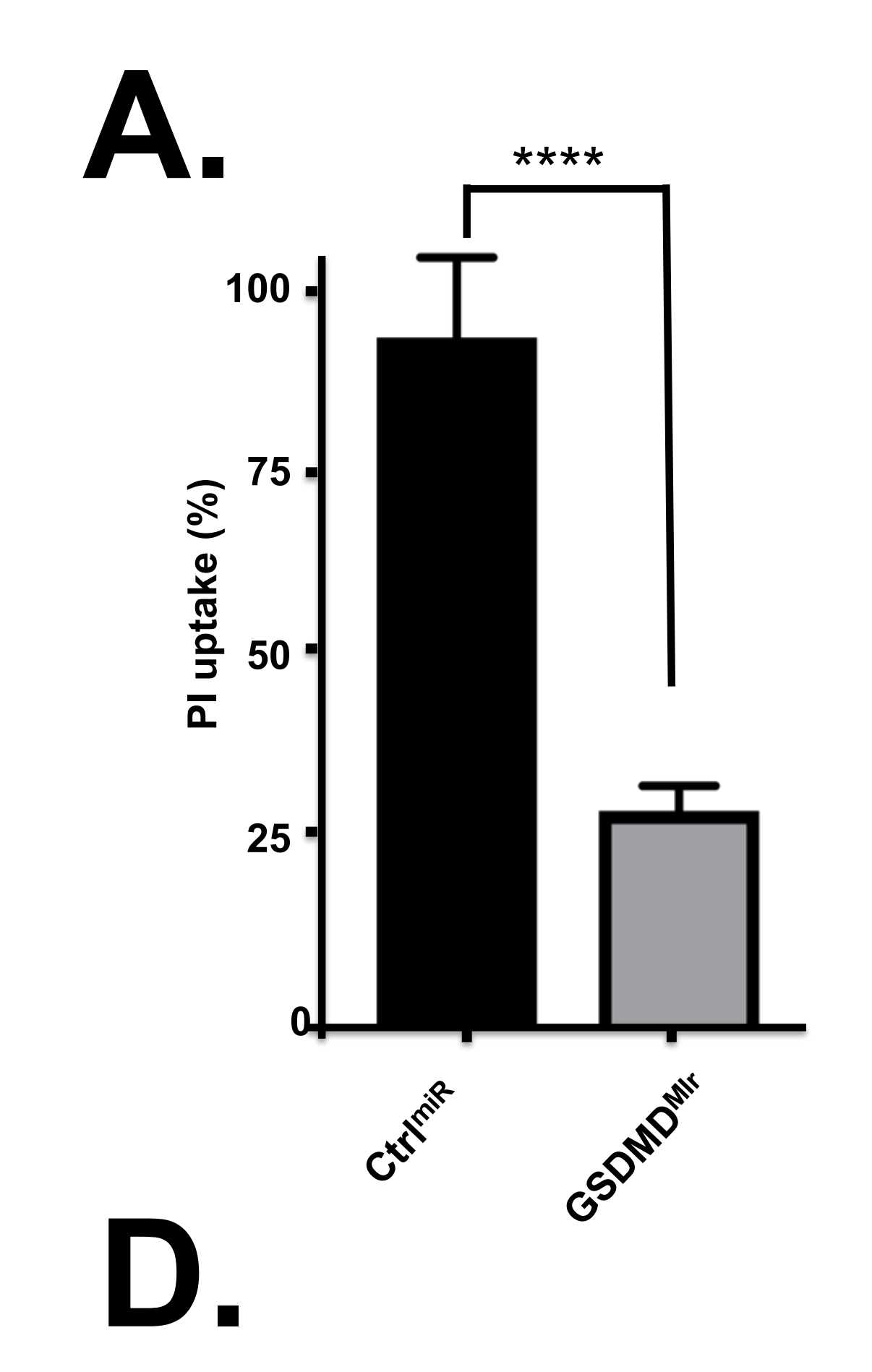

B.
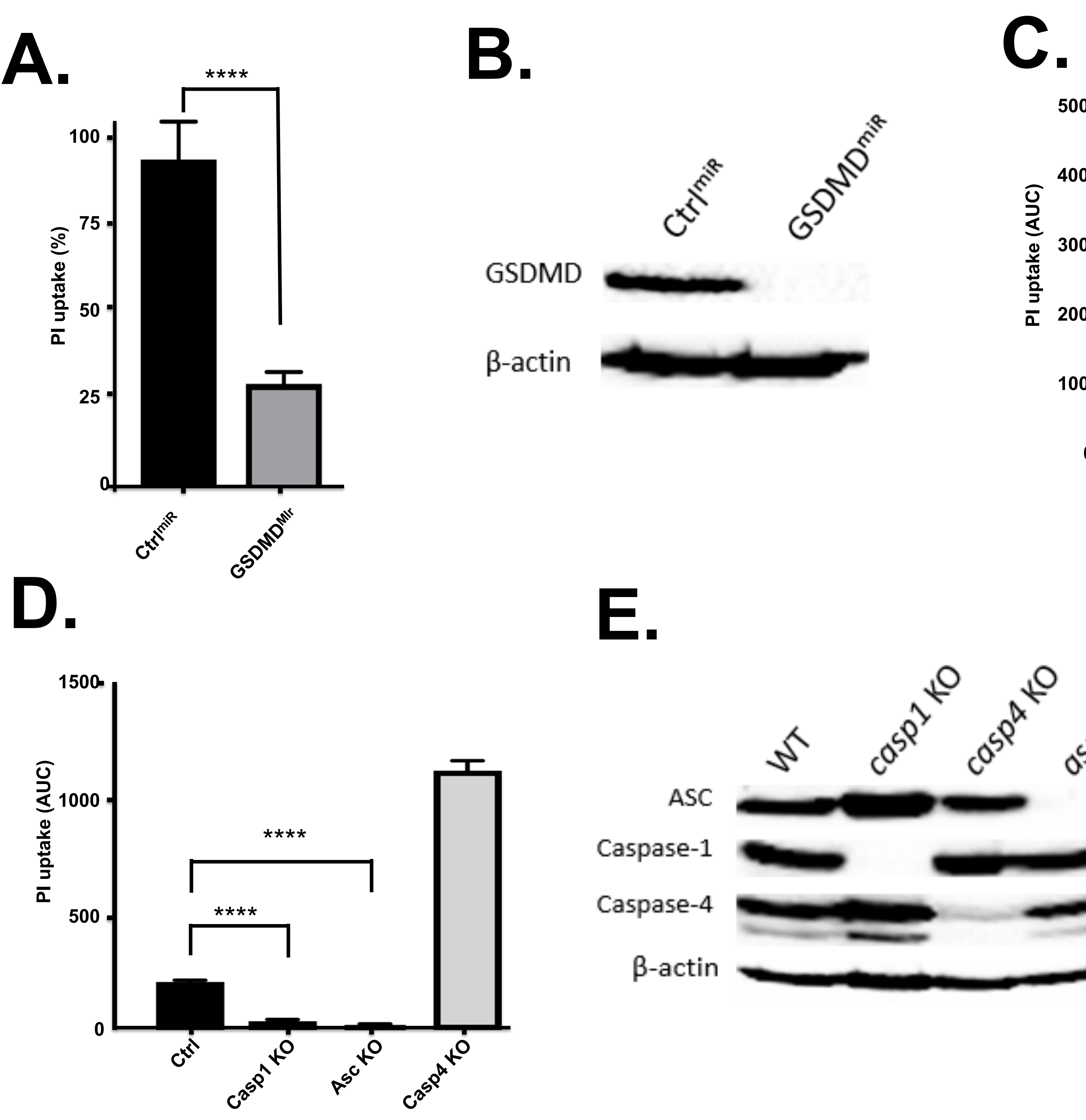

E.

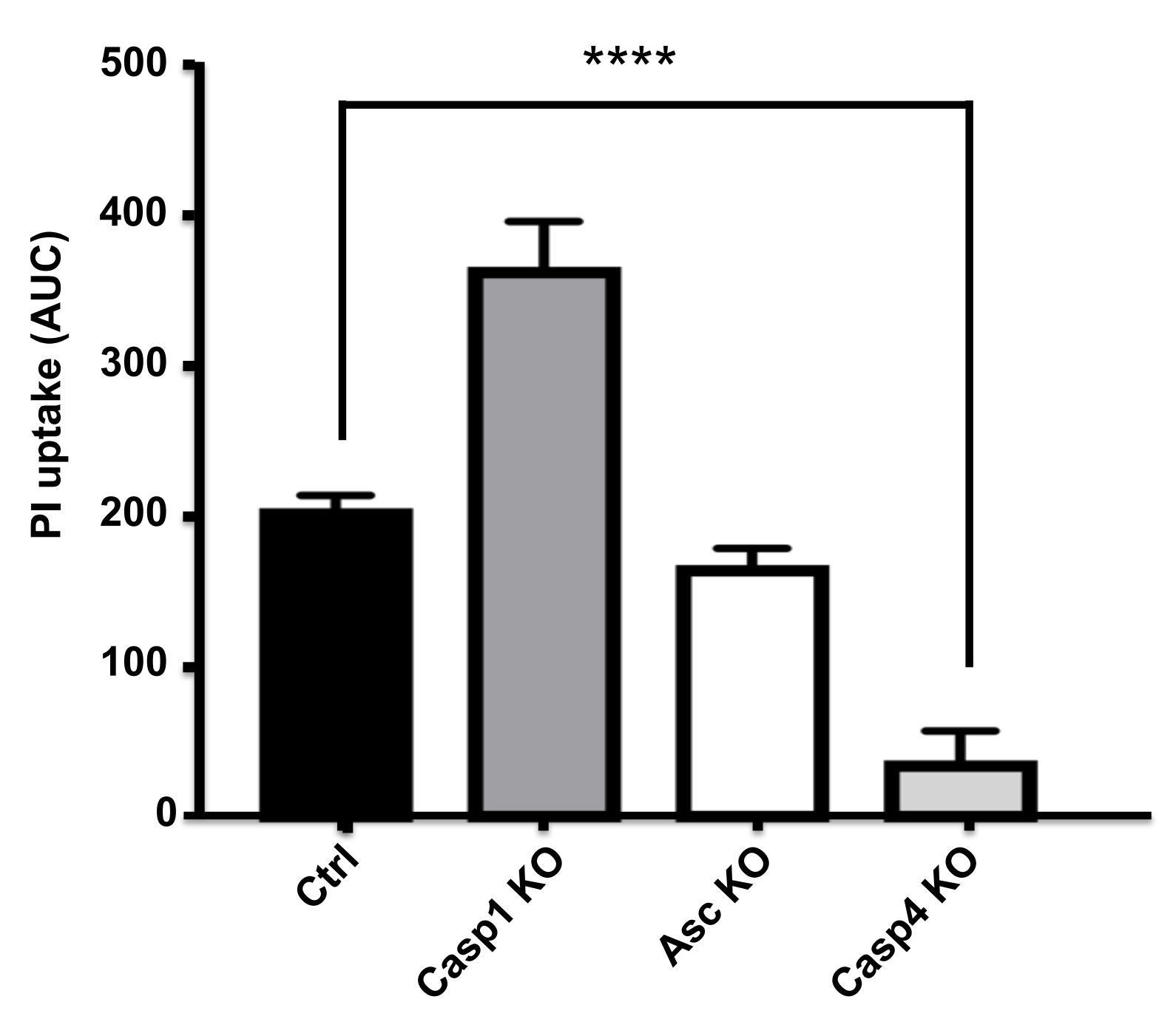

F.
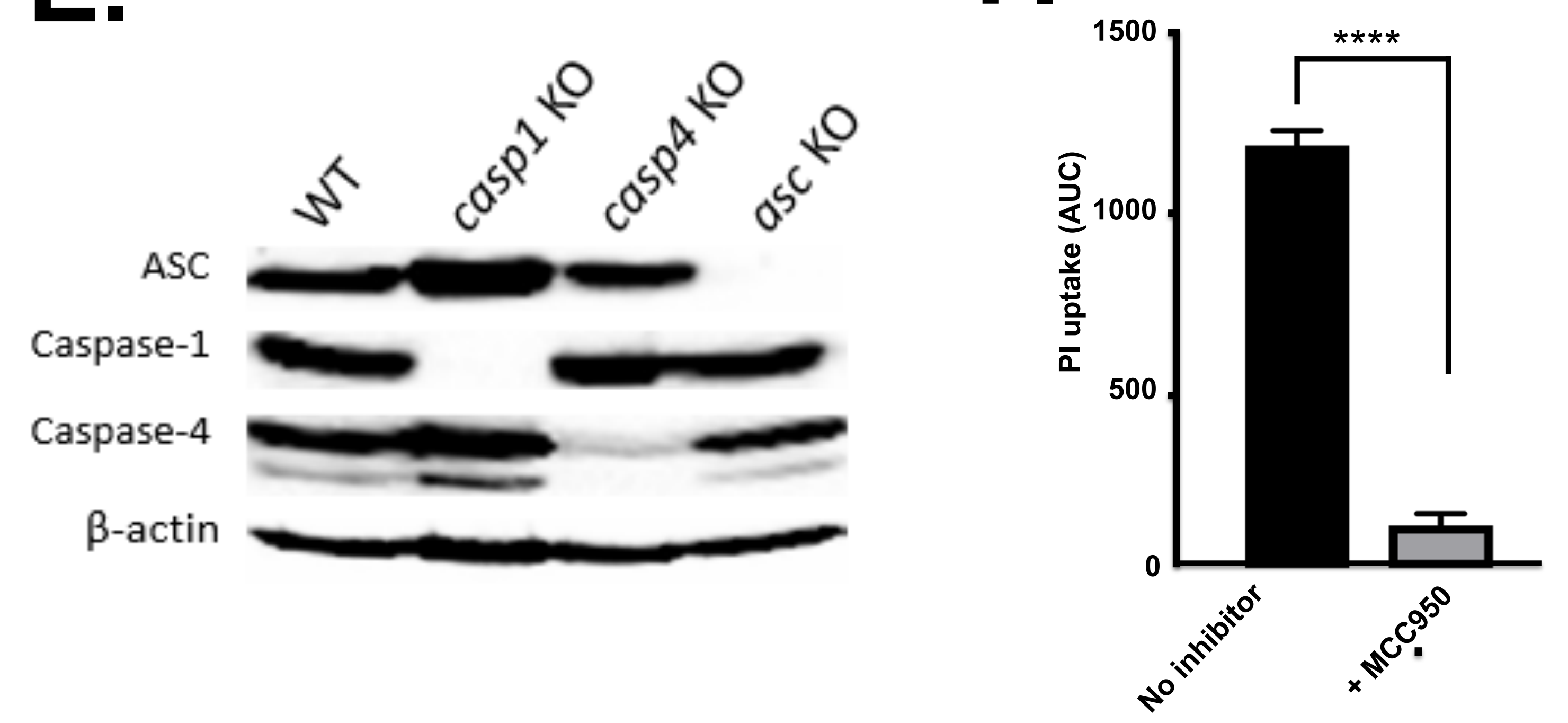

Figure S2 
A. B.
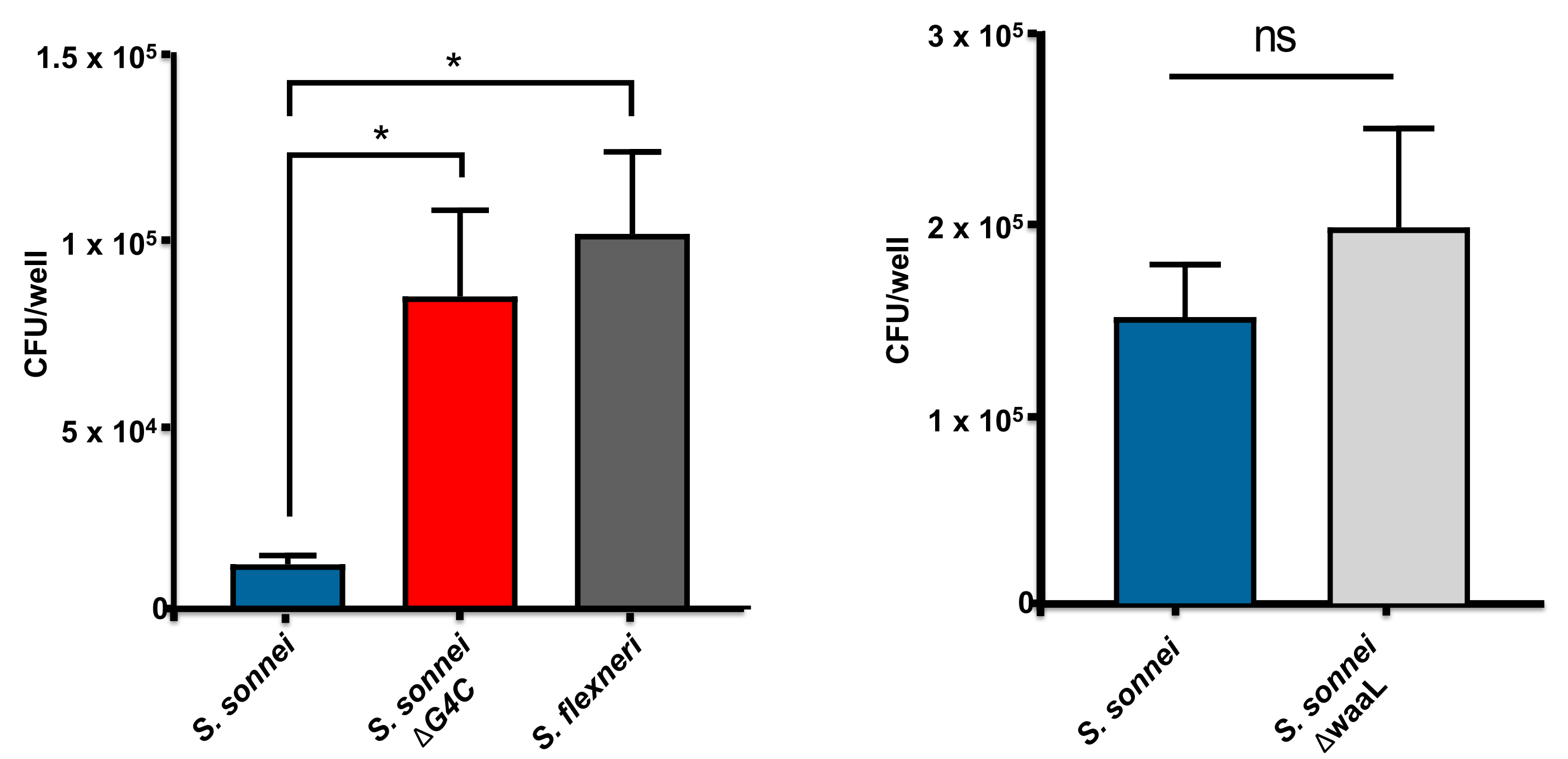

C.

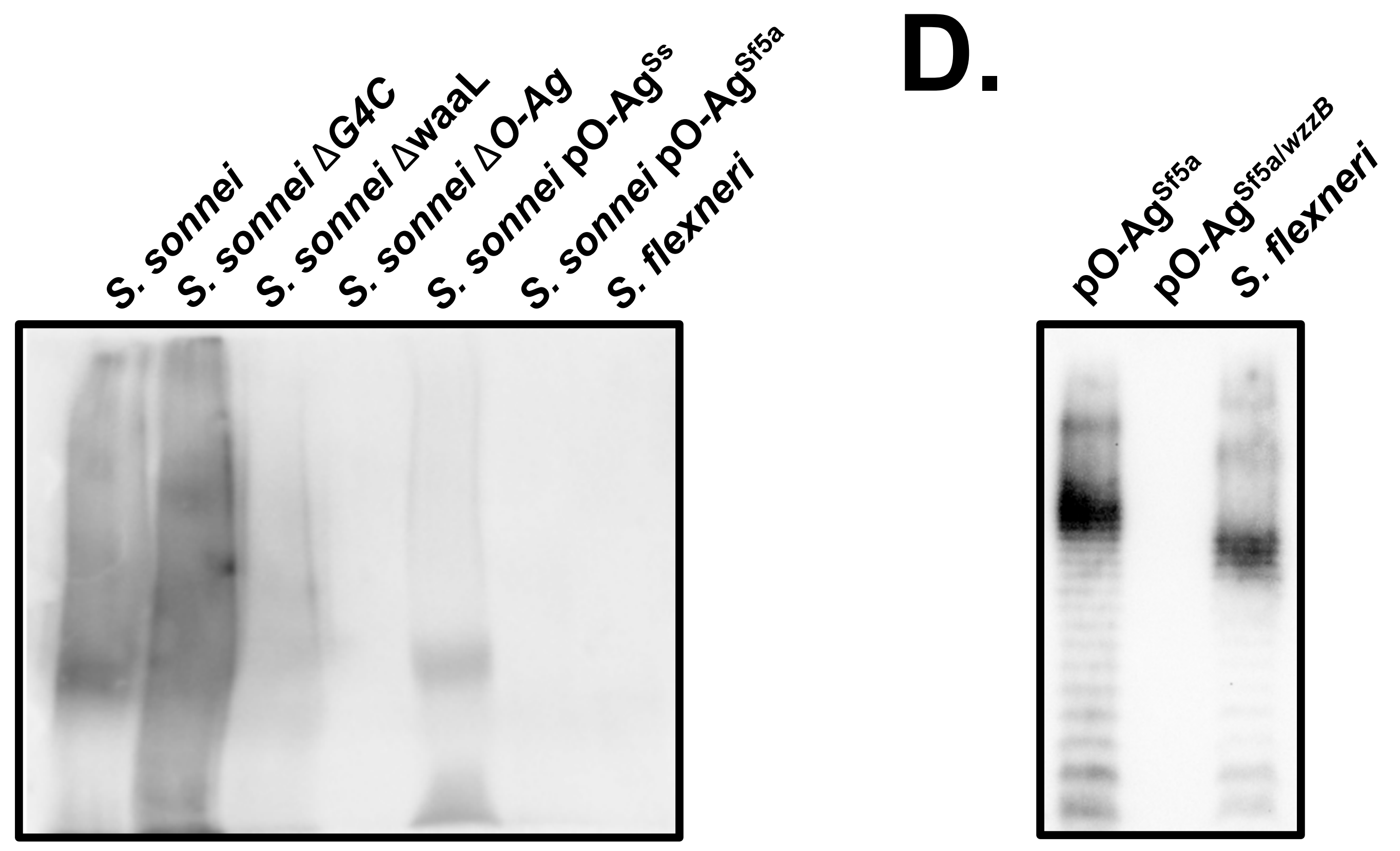

Figure S3 